\title{
Estimating Viability of Gopher Tortoise Populations
}

\section{US Army Corps

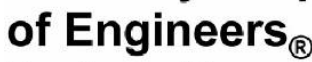
Engineer Research and Development Center

Tracey D. Tuberville, J. Whitfield Gibbons, and Harold E. Balbach

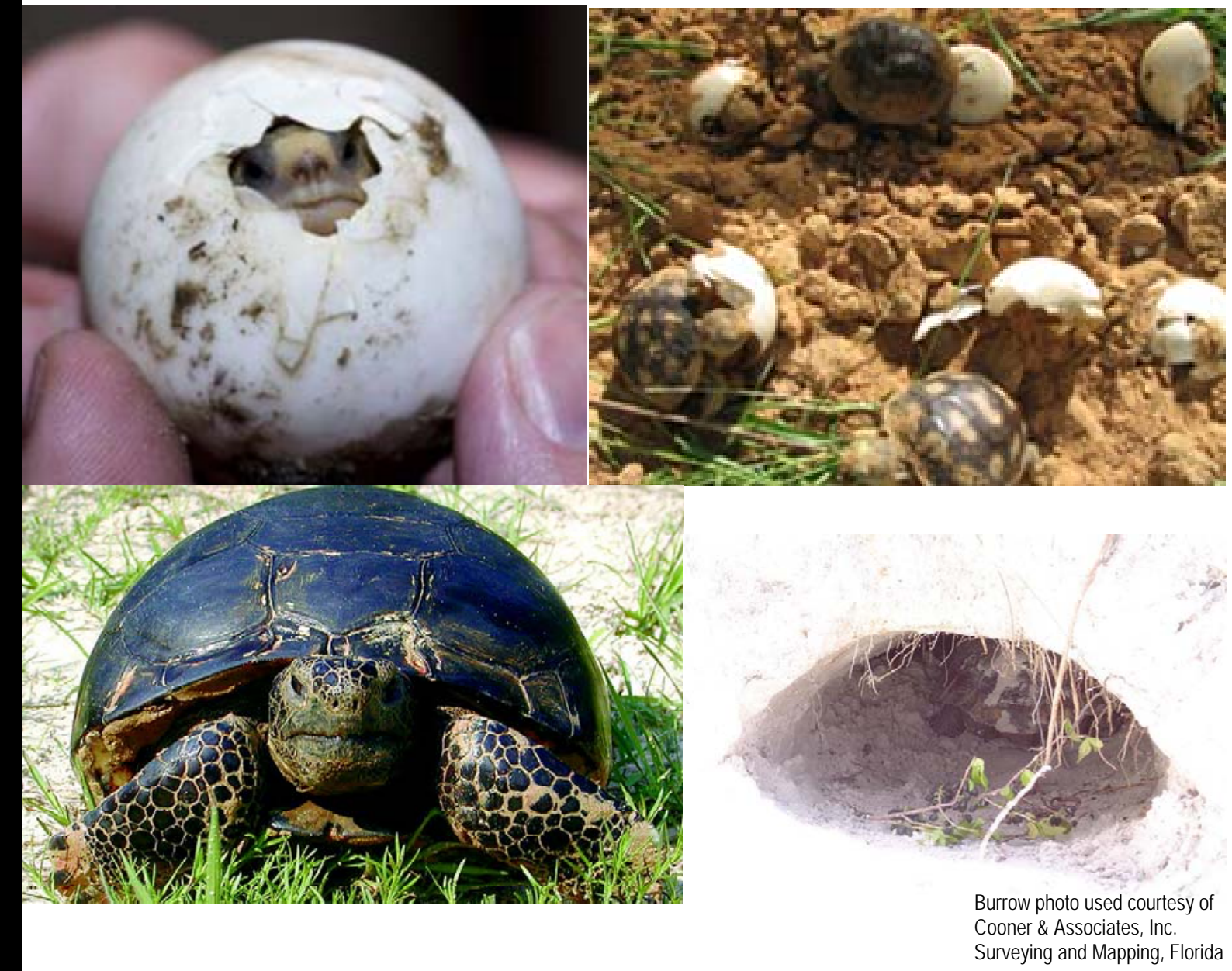




\section{Estimating Viability of Gopher Tortoise Populations}

Tracey D. Tuberville and J. Whitfield Gibbons, University of Georgia

Savannah River Ecology Laboratory

Aiken, SC 29802

Harold E. Balbach

Construction Engineering Research Laboratory (CERL)

U.S. Army Engineer Research and Development Center 2902 Newmark Dr.

Champaign, IL 61822

Final Report

Approved for public release; distribution is unlimited.

Prepared for U.S. Army Corps of Engineers

Washington, DC 20314-1000

Under Work Unit F61GB7 


\begin{abstract}
Although the gopher tortoise (Gopherus polyphemus) is widespread across the southeastern United States where conditions are favorable, the species is in serious decline. While biologists and land managers might consider any of a variety of in-situ and ex-situ management options when determining how best to manage individual populations, there are no good decision tools for evaluating or predicting whether the existing population is viable in the long term. This study developed demographic models for gopher tortoise populations and has proposed to use those models to predict outcomes for a variety of population conditions and management scenarios. The major limiting factor in the development of models was the availability of complete lifehistory data. Because of its demonstrated importance in population stability in other turtle species, adult survivorship was assumed to be high in all model scenarios. However, reproduction and survivorship of other life stages varied among simulations. The model results demonstrated that, of the parameters manipulated, hatchling survivorship was the most critical life history stage because of the very small likelihood that hatchlings survive to their second year. Any management actions that increase hatchling success are likely to increase population viability significantly.
\end{abstract}

DISCLAIMER: The contents of this report arenot to beused for advertising, publication, or promotional purposes. Citation of trade names does not constitute an official endorsement or approval of the use of such commercial products. All product names and trademarks cited are the property of their respective owners. The findings of this report are not to be construed as an official Department of the Army position unless so designated by other authorized documents. 


\section{Table of Contents}

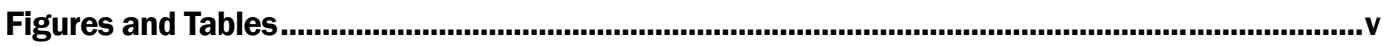

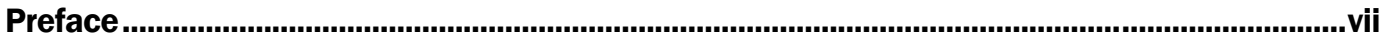

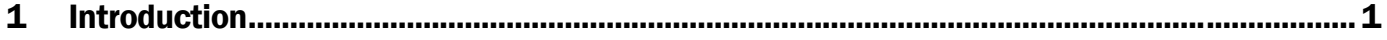

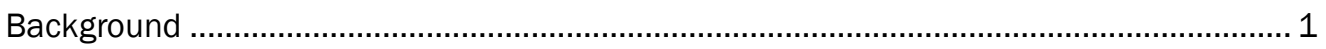

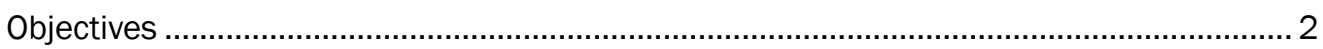

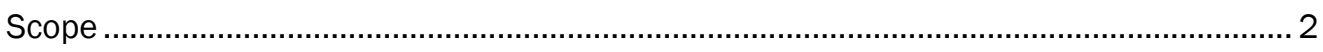

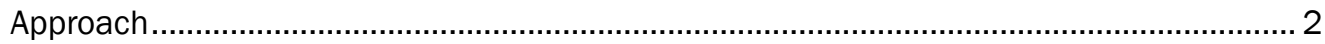

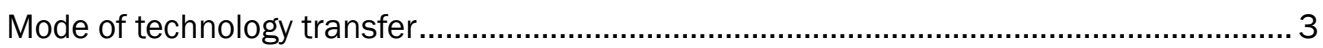

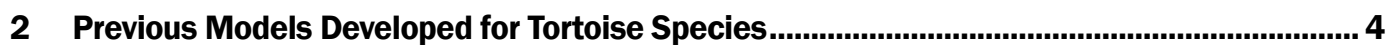

Utility of population modeling for managing populations of turtle species .......................... 4

Previous population models for Gopherus .................................................................... 5

Gopherus agassizii - Population Viability Analysis (PVA) for western Mojave Desert

(Doak et al. 1994)................................................................................................ 5

Gopherus polyphemus - Survival characteristics of small populations (Cox 1989)............... 6

Gopherus polyphemus - Estimating population viability (Cox et al. 1987)............................ 6

Gopherus polyphemus - PVA of relocated populations (Seigel and Dodd 2000)................... 7

Gopherus polyphemus - PVA for Florida (Miller et al. 2001) .............................................. 8

3 Baseline Model for Naturally-Occurring Gopher Tortoise Populations ................................... 9

How the current model differs from previous models....................................................... 9

Limitations of the model ............................................................................................. 10

Parameter selection and model development ............................................................ 11

Baseline model parameters for naturally-occurring populations......................................... 11

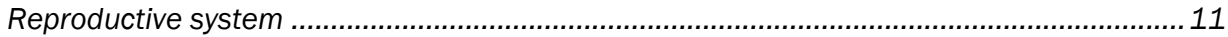

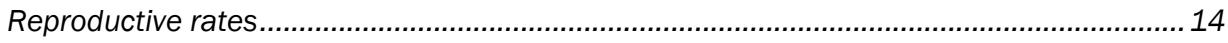

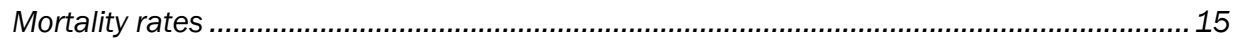

Mate monopolization (\% of males in breeding pool - 100\%) .............................................. 16

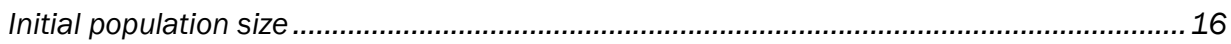

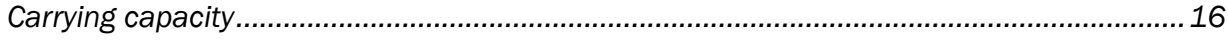

\section{Effects of Geographic Location, Habitat Quality, and Initial Gopher Tortoise}

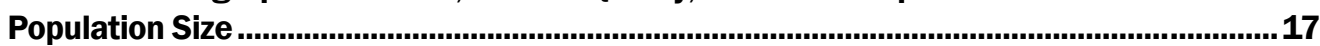

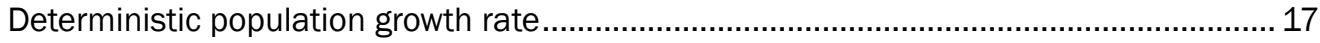

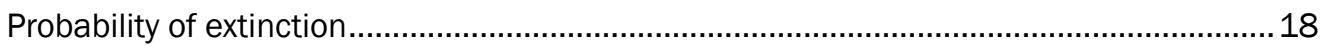

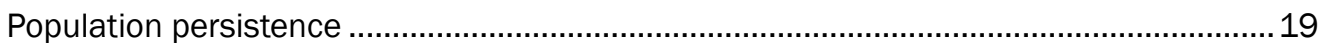

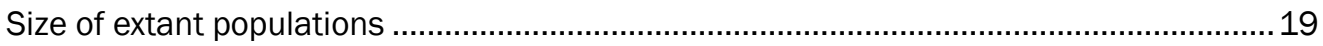

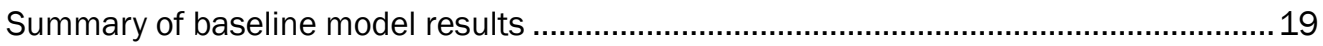

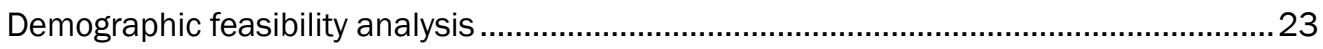

Demographic sensitivity analysis results ....................................................................... 25

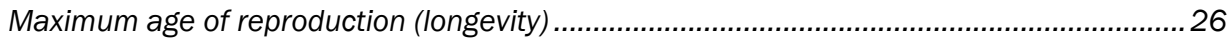

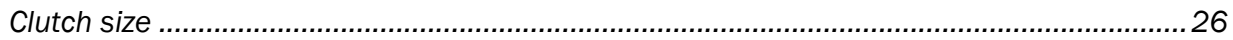




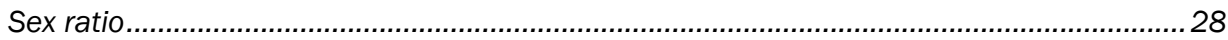

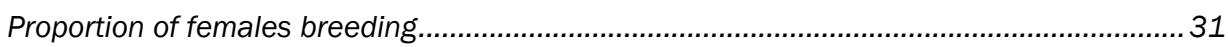

Comparative survivorship of immature stages.............................................................. 31

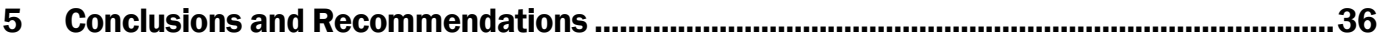

References......................................................................................................................................38

Acronyms and Abbreviations .............................................................................................43

Appendix A: Parameter estimates used in the VORTEX ${ }^{\circledR}$ baseline model for gopher tortoise populations ............................................................................................................44

Report Documentation Page..........................................................................................................48 


\section{List of Figures and Tables}

\section{Figures}

1 Geographic extent of the gopher tortoise, with the south, central, and peripheral portions of the range indicated

2 Deterministic population growth rate of simulated gopher tortoise populations in optimal (black bars) and marginal (gray bars) habitat conditions in different regions within the geographic range

3 Probability of extinction within 200 years for simulated gopher tortoise populations of different initial population sizes based on the population's location within the range and habitat conditions. (Note: $x$-axis scale is not linear.)........... 18

4 Probability of extinction within 100 years for simulated gopher tortoise populations of different initial population sizes based on the population's location within the range and habitat conditions. (Note: $x$-axis scale is not linear.).

5 Population persistence (or mean time to extinction) for simulated gopher tortoise populations of different initial population sizes based on the population's location within the range and local habitat conditions. Graphs plateau at 200 years, the maximum simulation duration possible. (Note: $x$-axis scale is not linear.)

6 Size of populations extant after 200 years based on their initial population size, location with the geographic range (south, central, periphery) and habitat quality (optimal, marginal). (Note: $x$-axis scale is not linear.)

7 Size of populations extant after 100 years based on their initial population size, location with the geographic range (south, central, periphery) and habitat quality (optimal, marginal). (Note: $\mathrm{x}$-axis scale is not linear.)

8 Population growth rates (top), probability of extinction within 200 years (center), and mean time to extinction (bottom) as a function of maximum age of reproduction.

9 Population growth rates (top), probability of extinction within 200 years (center), and mean time to extinction (bottom) as a function of mean clutch size. (The baseline clutch size is 6.75 eggs.)

10 Population growth rates (top), probability of extinction within 200 years (center), and mean time to extinction (bottom) as a function of sex ratio (\% females in initial population)

11 Population growth rates (top), probability of extinction within 200 years (center), and mean time to extinction (bottom) as a function of proportion of females breeding.

12 Population growth rates (top), probability of extinction within 200 years (center), and mean time to extinction (bottom) as a function of variation in hatchling, yearling and juvenile mortality rates from baseline model.

13 Population growth rates (top), probability of extinction within 200 years (center), and mean time to extinction (bottom) as a function of small variation in hatchling mortality rates from baseline model (baseline survivorship $=4 \%$ ) 


\section{Tables}

1 Ages at first reproduction for males and female gopher tortoises under different combinations of geographic location and habitat quality................................................... 13

2 Proportion of females breeding, as specified in this model, based on habitat quality.

3 Mean clutch size, as specified in this model, as a function of location within

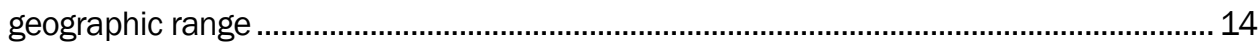

$4 \quad$ Stage-specific mortality rates as specified in our model ................................................. 15

5 A summary of demographic values that produced the most stable population of gopher tortoises in the demographic analysis, and the resulting measures of

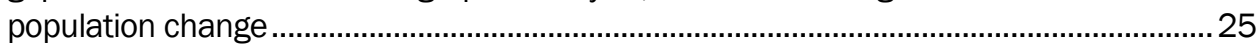

A1 Parameter estimates used in the VORTEX ${ }^{\circledR}$ baseline model for gopher tortoise populations.

A2 Stable age distribution calculated by VORTEX ${ }^{\circledR}$ for an initial population of 100 individuals.

A3 Manipulations of the baseline model for demographic sensitivity testing. The simulated population has an initial size of 100 individuals and occurs in optimal habitat in the central range. (The original parameter values used in the baseline model are represented by “B.") 


\section{Preface}

This study was conducted for the Army Chief of Staff for Installation Management (ACSIM) under Project 40162720A896, "Base Facilities Environmental Quality; work Unit F61GB7, "Rangewide Gopher Tortoise Conservation." The technical monitor was Scott Belfit, DAIM-ED

The work was completed under the direction of the Ecological Process Branch (CN-N) of the Installations Division (CN), Construction Engineering Research Laboratory (CERL). The CERL Principal Investigator and contract monitor was Dr. Harold Balbach. The modeling analyses and preliminary report preparation were performed by Dr. Whit Gibbons and Tracey Tuberville, University of Georgia. Savannah River Ecology Laboratory (SREL), Aiken, SC under Cooperative Ecosystem Studies Unit agreement No. W9132T-06-2-0019.Alan B. Anderson is Chief, CN-N, and Dr. J ohn T. Bandy is Chief, CN. The associated Technical Director was Dr. William D. Severinghaus. The Director of CERL is Dr. Ilker Adiguzel.

CERL is an element of the U.S. Army Engineer Research and Development Center (ERDC), U.S. Army Corps of Engineers. The Commander and Executive Director of ERDC is COL Gary E. J ohnston, and the Director of ERDC is Dr. J ames R. Houston. 


\section{Introduction}

\section{Background}

The gopher tortoise (Gopherus polyphemus) is considered to be declining throughout its range (Smith et al. 2006) and is Federally listed in the western portion. The U.S. Fish and Wildlife Service has also been petitioned to list the remaining populations (Save Our Wild Scrub et al. 2006). The Florida Fish and Game Commission estimates that-in that state alone-74,000 gopher tortoises have been impacted by incidental take permits issued to developers in the past 14 years (J. Berish, pers. comm.). Although the estimate does not necessarily represent number of tortoises actually killed (because some were relocated), the extent of the loss is alarming and is not sustainable in a long-lived species such as the gopher tortoise in which population stability is contingent on high adult survivorship (Congdon et al. 1993). Other estimates suggest that informal relocations not requiring permits may have affected even more animals $(R$. Ashton, pers. comm.) Ironically, many sites that are permanently protected and have appropriate or restorable habitat, apparently no longer support "viable" tortoise populations (McCoy et al. 2006).

A regional goal identified at recent regional workshops ${ }^{1}$ was to improve management of the tortoise region-wide so that further Federal listing of the gopher tortoise in the eastern portion of its range would no longer be necessary. However, there are currently no good decision tools for evaluating or predicting when an existing tortoise population is likely to be selfsustaining, or when management intervention is needed for a specific population. In-situ habitat management and protection are the preferred conservation tools for promoting viable populations of any wildlife species. However, habitat-based approaches may not always be the most effective strategy or even be possible in some circumstances (e.g., at locations with severely-degraded habitats, small habitat patches, or sites with remnant tortoise populations, etc). This study was undertaken to develop demographic models that may be used to evaluate the long-term viability of gopher tortoise populations under varying initial population conditions and management scenarios, and to explore whether better habitat manage-

\footnotetext{
1 Fall-Line Sandhills Workshop, Savannah River Ecology Laboratory (SREL), March 2005; Interagency Gopher Tortoise Habitat (Eastern population) Workshop, Fort Gordon, June 2005.
} 
ment is likely to improve persistence of populations deemed as unlikely to be viable under current conditions.

\section{Objectives}

The objectives of this study were to:

1. Develop appropriate demographic representations of gopher tortoise populations based on life history parameters derived from the literature, unpublished data, and expert opinion.

2. Use this information in the Vortex ${ }^{\circledR}$ population viability analysis software environment to model significant elements of population viability for this species and to predict outcomes (e.g., population persistence times and extinction probabilities) for different initial population conditions and management scenarios.

3. Develop a decision tool to assist land managers in evaluating the best options for managing declining or other "at-risk" populations.

4. Compile relevant data to be used in the preparation of biological assessments (BAs) and biological opinions (BOs) related to Army training activities potentially impacting gopher tortoise populations, and for endangered species management plans (ESMPs), integrated natural resources management plans (INRMPs), and in the preparation of ecological risk assessments involving training or equipment testing where the tortoise is present.

\section{Scope}

This study is intended to apply to all tortoise populations in the Southeastern states, assuming appropriate selection of parameters. Although general findings should be broadly applicable to all populations, it is not proposed that the Vortex ${ }^{\circledR}$ model be applied to each local population. Still, the application of the the Vortex ${ }^{\circledR}$ model is encouraged if local data are more complete than the estimates readily available for this study.

\section{Approach}

1. A literature review was completed to investigate previous models developed for tortoise species

2. Gopher tortoise population models were constructed using the software program Vortex ${ }^{\circledR}$ (Lacy et al. 2005). The results of these models were presented in two parts:

a. Part 1- baseline model results, and 
b. Part 2 - demographic sensitivity testing.

3. Findings were summarized and recommendations made for the application of this model to the conservation and management of individual populations of gopher tortoises.

\section{Mode of technology transfer}

It is anticipaed that the results of this work will be used to develop guidance for land managers and threatened and endangered species (TES) program managers to assist them in managing the tortoise populations on lands for which they are responsible. Population viability information will be published in the scientific journals, and through formal laboratory publication. This report will be made accessible through the World Wide Web (WWW) at URL: http://www.cecer.army.mil 


\section{Previous Models Developed for Tortoise Species}

A number of population models have been used to address conservation issues facing turtles. This chapter summarizes other models developed for tortoise species.

\section{Utility of population modeling for managing populations of turtle species}

Population models, which can be a powerful tool for managing turtle populations, have been used to:

1. Rank relative threats to specific populations

2. Evaluate effects of proposed management actions or regulations

3. Determine the demographic or ecological variables that have greatest influence on extinction risk

4. Identify information gaps and research priorities.

The major limiting factor in the development of realistic population models is the availability of complete life-history data. Congdon and colleagues developed some of the first and most complete life tables for individual turtle species, based on over 30 years of intensive data collection on Emydoidea blandingii and Chelydra serpentina (Congdon et al. 1993, 1994). However, given that such comprehensive data are generally not available for most species, most existing models have used data from multiple populations over a shorter time span per population, compiled information from the literature, or have accepted (or even incorporated) uncertainty in their model.

For those species lacking complete life history data, population models can still be useful for managing populations. For example, Rivera and Fernandez (2004) and Horne et al. (2003) conducted threat analyses for specific populations of Emys orbicularis and Graptemys flavimaculata, respectively, to develop management plans for those populations. Pedrona et al. (2004) evaluated the likely effectiveness of exchanging individuals between captive and wild populations of Geocheloneyniphora to simulate a metapopulation. Heppell et al. (2005) used population models to evaluate the potential effects of turtle excluder devices on reducing by-catch of sea turtles. Heppell et al. (1996) used models to explore the potential utility of 
head-starting as a management tool. Heppell (1998) used life table data from populations of several different species and conducted an elasticity analysis to look for similarities across species in those demographic variables most likely to determine whether populations continue to decline.

\section{Previous population models for Gopherus}

Several studies have specifically modeled population dynamics of the gopher tortoise or its western counterpart, the desert tortoise. Although their life histories are not identical, the two species have many ecological similarities, face many of the same management issues, and both are often the focus of translocation efforts. The following sections discuss each population model, highlighting major findings relevant to this modeling effort.

\section{Gopherus agassizii - Population Viability Analysis (PVA) for western Mojave Desert (Doak et al. 1994)}

Doak et al. (1994) developed a population model to evaluate the potential impact of the proposed expansion of Fort Irwin on Gopherus agassizii populations. The model is stage-based (meaning that demographic characteristics are a function of developmental stage rather than age). It is a regional model for the western Mojave Desert (rather than for a single population) and is based on periodic census data collected from eight Bureau of Land Management sites, with two to four census periods per site during the period of 1979-1989. The model is based on data from females only and demographic rates are assumed to be constant over the time interval between censuses. The model was designed to specifically investigate temporal variability in demographic rates and the correlation among demographic rate responses to environmental variables.

Doak et al. (1994) concluded that the models were most sensitive to changes in survival rates, particularly of subadult females just entering reproduction, and that better survival estimates were needed. They also determined that both the temporal variability in demographic rates and the correlated responses among demographic rates resulted in greater variability of population growth rates, dramatically increasing uncertainty about how accurately the predicted short-term population trends reflect long-term fate of desert tortoise populations. 


\section{Gopherus polyphemus - Survival characteristics of small populations (Cox 1989)}

Cox (1989) conducted a population viability analysis to determine the importance of small populations of gopher tortoises and their relative vulnerability to extinction under harsh, moderate, and favorable conditions. Both deterministic and stochastic models (that is, without and with demographic and environmental variation incorporated) were constructed for initial population sizes of 6, 10, 14, 20, 30, and 40 individuals. Both adultonly and mixed (50 percent adults, 50 percent subadult) populations were simulated. For each scenario, 40 simulations were conducted and simulation duration was 200 yrs. The potential effects of competition, densitydependence, and immigration were not modeled, but effects of inbreeding depression were incorporated into the model. The selected demographic parameters were based on data from Florida and south Georgia.

Based on the persistence of populations of 20 or more individuals for more than 100 years, Cox concluded that 20 individuals was a pivotal number under the conditions modeled and that even small populations can contribute to the species' long-term persistence. The model was most sensitive to changes in adult and subadult survivorship, then secondarily by additionally increasing either survivorship of other classes or fecundity. As might be predicted, model outcomes were particularly sensitive to stochasticity when population sizes were small.

Although the model is based on data from naturally-occurring populations, Cox notes the potential application of the model results to relocation and emphasizes that the model would need to be modified to reflect changes in tortoise behavior (e.g., dispersal) and demography resulting from relocation. For example, mixed-stage populations performed slightly better than adult-only populations, suggesting the value in relocating individuals from all stages in the population. Also, populations of relocated tortoises may exhibit higher mortality and/ or dispersal rates than those reported for naturally occurring populations, and would likely dramatically affect model outcome.

\section{Gopherus polyphemus - Estimating population viability (Cox et al. 1987)}

The Cox et al. (1987) model is similar to the model presented in Cox 1989, but models populations over a wider set of initial population sizes (10- 150 tortoises) and with a more complex demographic structure (10 percent juveniles, 30 percent subadults, and 60 percent adults). Minimum viable 
population sizes (defined by Cox as minimum initial population size with at least 90 percent probability of surviving for at least 200 years) were calculated under harsh (little or no management), moderate, and favorable conditions to simulate the effects of management. The different management conditions were modeled by varying survival of all age classes and fecundity among the scenarios.

Even large populations had difficulty persisting under harsh conditions and an estimated minimum of 310 tortoises was needed for the population to have at least 50 percent chance of surviving for 200 years. Under moderate conditions, even relatively large populations persisted for longer periods than under harsh conditions, but still did not meet desired management goals. At least 130-150 tortoises were needed to persist under moderate conditions. Under favorable conditions, even small populations of at least 40-50 animals performed well.

\section{Gopherus polyphemus - PVA of relocated populations (Seigel and Dodd 2000)}

The purpose of the Seigel and Dodd (2000) model was to examine how retention rates of translocated adult gopher tortoises could influence shortterm population viability. Because gopher tortoises are long-lived animals, the authors note that the species is vulnerable to changes in survivorship (or site fidelity) of adults and older juveniles, which might be expected to occur following translocation.

Seigel and Dodd modeled populations initially composed of 50 adult animals for a 30 year period, with age at maturity, first year survivorship, and all reproductive parameters based primarily on data collected from Mississippi and Louisiana at the western limit of the species' range. Note that the data were collected from populations within the Federally listed range. Adult survivorship was varied among scenarios to reflect post-translocation annual retention rates of 80, 85, 90, and 95 percent, which were held constant for the duration of the simulation. For each scenario, they ran 10 simulations and calculated the average number of turtles remaining after 30 years and the probability that the modeled population would go extinct during the simulation period.

The resulting model predicted rapid population decline except when retention rates of adult relocated tortoises were very high (at least 90 percent). The authors note that their model is based on the assumption that retention rates remain low over the simulation period rather than increasing 
over time to levels more representative of naturally-occurring populations. They assert that the short-term nature of post-relocation monitoring, when conducted at all, precludes altering that assumption. However, as noted by Ashton and Burke (2007) and Tuberville et al. (2008, in review), data from more extensive post-translocation monitoring are now available.

\section{Gopherus polyphemus - PVA for Florida (Miller et al. 2001)}

Miller et al. (2001) proposed to investigate the probability that gopher tortoises are likely to become extirpated from the state of Florida over the next 100 years, whether considering all known populations within the state or considering only populations on public lands. The model also incorporates regional variation of life-history parameters within Florida and the potential impacts of Upper Respiratory Tract Disease (URTD) on population fate. Parameters used in the model are based on data collected in Florida and southern Georgia, and the initial population sizes in the model were chosen to encompass population estimates from 294 sites in Florida. Construction of the model within Vortex ${ }^{\circledR}$ was guided by expert opinion. As has been observed with other models for gopher tortoises and other turtle species, model outcomes were most sensitive to survivorship of adult females and juveniles. Effects of regional variation in age at maturity indicate that more northerly populations are less buffered against uncertainty in model parameters and are less able to withstand additional sources of mortality (e.g., Upper Respiratory Tract Disease). So while the statewide "population" was deemed not at risk of extinction, Miller et al. (2001) note individual populations may be at risk under certain conditions, especially where anthropogenic factors are combined with the unpredictability associated with normal demographic or stochastic variability. The authors concluded that, under favorable conditions, the models indicate that even populations as small as 50 individuals can contribute to the conservation of the species. 


\section{Baseline Model for Naturally-Occurring Gopher Tortoise Populations}

The methodological approach used to derive and apply this model was to develop a series of simulated populations broadly categorized in terms of intrinsic and extrinsic characteristics rather to develop a such a series based on site-specific data from any one population. For populations without pre-existing information, the models should help prioritize the data that need be collected to effectively manage the population of interest. In addition, the current model, as developed and applied here, draws on data from throughout the range of the gopher tortoise rather than just the core of the species' range, which has been the focus of most previous models. Model results were reported in terms of final mean population sizes, population persistence times, and probabilities of extinctions, which are all important model output and which also make it possible to compare these results to those of previous models. However, it is proposed here that focusing on extinction risk, per se, without considering trends can mask negative population trends that signal a need for management intervention. This is especially important where the time limit of the model (200 years) represents only a handful of tortoise generations.

\section{How the current model differs from previous models}

Gopher tortoises will likely continue to suffer habitat loss under the pressures of development. Meanwhile, populations on some protected lands have experienced declines and are unlikely to recover without improved, more intensive, management (McCoy et al. 2006). Therefore, there is a need to be able to evaluate the viability of individual populations, rank the populations most appropriate for in-situ protection, and determine if nonviable populations are more likely to contribute to conservation of the species through augmentation or translocation.

The target audience and end user for the current model is the individual land manager who is charged with evaluating and managing discrete tortoise populations for viability. Therefore, the focus is on individual populations, as found on one parcel or in one landscape rather than a regional or state-wide collection of populations. This study attempted to broadly categorize populations in terms of intrinsic and extrinsic characteristics that land managers should be able to apply to their population of interest based 
on data or information that either is likely already available for the population, could be relatively easily collected, or would be part of a tortoise monitoring program. For sites without pre-existing information, the models should help prioritize the data that need be collected to effectively manage the population of interest.

In addition, the current model "database" draws on data from throughout the range of the gopher tortoise rather than just the core of the species' range, which has been the focus of most previous models. Finally, greater emphasis is placed here on population growth rates (lambda) predicted under different model scenarios. This study reported on final mean population sizes, population persistence times, and probabilities of extinctions, which are all important model output, and which also make it possible to compare these results to those of previous models. However, it is proposed here that focusing on extinction without considering lambda values can mask negative population trends that signal a need for management intervention, especially with this long-lived species. Lambda is also a tangible variable that can be:

1. Calculated over specific monitoring intervals from field-collected data

2. Used to compare the relative effectiveness of different management strategies

3. Compared to lambda estimated by the model to evaluate model validity.

This is especially important where the time limit of the model (200 years) represents only a handful of tortoise generations, rather than the 200 generations typically assumed when modeling some of the most common mammal and bird species for this time period.

\section{Limitations of the model}

Like all other population-based models for the species, this model is based on incomplete knowledge of certain aspects of gopher tortoise life history and must be qualified accordingly. Comprehensive empirical data are completely lacking for some parameters, such as longevity. In addition, for those parameters for which data are available, estimated values are an amalgamation of values reported from multiple sites distributed across a wide geographic area and that may vary in habitat quality. Because life history trait values are likely to vary among populations and because different trait values were available from different study populations, the combination of parameters used here are not necessarily representative of any single population. In addition, many of the published data are based on rela- 
tively short-term studies that provide only a snapshot view of tortoise population dynamics. One must recognize the limitations of currently available data and use care in applying the results of this model, which will necessarily require updating and re-interpretation as more complete data become available.

However, this model should adequately represent the current understanding of gopher tortoise life history based on the best available, though admittedly not precise, estimates for parameter values. Where appropriate, the broader literature on life histories of other turtle species was used to assist in predicting how life history trait values might vary under different model scenarios.

\section{Parameter selection and model development}

\section{Baseline model parameters for naturally-occurring populations}

The models described here were constructed using VORTEX ${ }^{\circledR}$, version 9.70 (Lacy et al. 2005), an individual-based model in which the fate of individual animals is monitored as they experience demographic and stochastic events that follow user-specified probabilities and distributions. VORTEX ${ }^{\circledR}$ is an age-based model in which survivorship and fecundity values vary with (and are specified according to) age rather than size or life stages. VORTEX ${ }^{\circledR}$ is able to model species with polygynous breeding systems and can easily simulate specific management scenarios such as catastrophic stochastic events, managed harvests, and translocation (Miller and Lacy 2005). VORTEX ${ }^{\circledR}$ was also used in the previous gopher tortoise simulations constructed by Miller et al. (2001) and Seigel and Dodd (2000), facilitating comparison of results among the various studies.

Listed below (and summarized in Appendix A, Table A1, p 45) are the parameters and their values as specified in VORTEX ${ }^{\circledR}$ for the current model. For each scenario, 100 simulations were run for 200 years, the maximum duration possible in VORTEX ${ }^{\circledR}$.

\section{Reproductive system}

Breeding system - Polygynous

Attempted matings with multiple mates within a breeding season has been noted for both male and female gopher tortoises (Boglioli et al. 2003; J ohnson et al. 2007). Multiple paternity of single clutches has also been documented (Moon et al. 2006; Tuberville 2008). 
Age at first reproduction

Site-specific factors (such as location within geographic range and habitat quality) presumably have systematic effects on tortoise growth and, as a result, on age at maturity (Landers et al. 1982; Mushinsky et al. 1994). Although length of the activity season (and opportunity for growth) varies with latitude, there is also evidence that growth rates among tortoises can vary significantly among local populations as a result of habitat quality (Aresco and Guyer 1999a; Mushinsky et al. 1994). Habitat quality can be manipulated through management; however, it is predicted that viability of populations in the more northerly portions of the species' range will be less resilient to marginal habitat conditions.

Ages at first reproduction are specified for the different combinations of geographic location (Figure 1) and habitat quality (Table 1). They are intended to reflect the average age at first reproduction, not the minimum age at which first reproduction has been reported. Each scenario assumed that males mature at a younger age than females (Landers et al. 1982; Mushinsky et al. 1994). The actual relationship may be one of attained body size rather than age, but varying age at first reproduction with location and site quality seems to be an adequate surrogate for size at first reproduction, which cannot easily be modeled using the selected software program.

Maximum age at reproduction (i.e., Longevity) - 60 years

There is no evidence to suggest that tortoises do not reproduce throughout their adult life. However, there are also no reliable data available for life span of gopher tortoises. Maximum age at reproduction was set at 60 years, as in Miller et al. (2001). How well this estimate reflects either historical or current conditions is unknown.

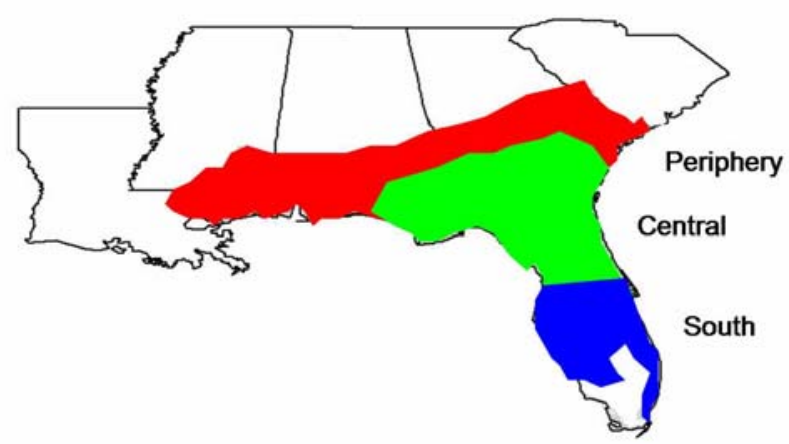

Figure 1. Geographic extent of the gopher tortoise, with the south, central, and peripheral portions of the range indicated. 
Table 1. Ages at first reproduction for males and female gopher tortoises under different combinations of geographic location and habitat quality.

\begin{tabular}{|l|c|c|c|}
\hline \multirow{2}{*}{ Location within geographic range } & \multirow{2}{*}{ Habitat quality } & \multicolumn{2}{|c|}{ Age at first reproduction } \\
\cline { 3 - 4 } & optimal & 13 & 12 \\
\hline South & marginal & 15 & 13 \\
\hline & optimal & 15 & 11 \\
\hline Central & marginal & 17 & 15 \\
\hline & optimal & 20 & 17 \\
\hline Periphery & marginal & 23 & 20 \\
\hline
\end{tabular}

Max number of progeny per year - 12

Although set at 10 in Miller et al. (2001), maximum number of progeny was set at 12 in this model. Clutch sizes of 11 or 12, while not common, do not appear to be an anomaly (Epperson and Heise 2003; Landers et al. 1980; Pike and Seigel 2006; Rostal and J ones 2002; Tuberville 2008). Due to nest failures (e.g., from predation) and less than 100 percent hatching success (e.g., from infertile eggs, early embryonic death), not all eggs will result in progeny. However, because nest and hatching success tend to vary stochastically as a result of extrinsic factors, it is more appropriate to incorporate them into the model elsewhere rather than here under maximum number of progeny (which is considered here as a constant intrinsic variable). Mean clutch sizes are specified separately in the model (see "Reproductive Rates," below, p 14).

Sex ratio at birth (in \% males) - 50\%

Although this ratio can vary from clutch to clutch as a function of nest temperature, it is assumed to be 50:50 for the population as a whole. In general, warmer nest temperatures produce more females and cooler temperatures produce more males, with temperatures at either extreme limiting hatching success (Burke et al. 1996; Demuth 2001). Theoretically, global warming could produce female-biased clutches; however, the potential effect of global warming on sex ratios of gopher tortoise cohorts is not well understood and, therefore, has not been incorporated into this model.

\section{Density-dependent reproduction}

This option was not activated for the model. However, density-dependent factors such as allee effects, which may occur in gopher tortoise populations (Boglioli et al. 2003; Guyer et al. 2006), could be incorporated in future models. 


\section{Reproductive rates}

Proportion of females breeding

The annual proportion of females breeding in a population may be greater (Rostal and J ones 2002) and presumably less variable in optimal, wellmanaged habitat than in marginal or unmanaged habitat. The parameter values specified in this model (Table 2) are substantially less optimistic than the values presented in Miller et al. (2001), but are based on data by Rostal and J ones (2002) and Smith et al. (1997).

Distribution of number of offspring per female per year - Normal distribution

The user can elect to specify the exact distribution of clutch sizes or to have the computer generate an approximation of the normal distribution based on a user-specified mean and standard deviation. Land managers are unlikely to have sufficient data to specify an exact distribution for clutch sizes from their population, but are likely to know the average clutch size of their population or a similar one. Therefore, the VORTEX ${ }^{\circledR}$ software was used to generate a normal distribution based on specified means and standard deviations in clutch sizes, which was varied among locations within the geographic range (Table 3). Model constraints assume that clutch sizes do not vary with age of female. Although this assumption may be violated, there are no data to estimate age-specific fecundity.

Table 2. Proportion of females breeding, as specified in this model, based on habitat quality.

\begin{tabular}{|c|c|c|}
\hline Habitat quality & $\%$ adult females breeding & E.V.* in \% breeding \\
\hline Optimal & 95 & 5 \\
\hline Marginal & 80 & 10 \\
\hline *E.V. = environmental variation (S.D.) \\
\hline
\end{tabular}

Table 3. Mean clutch size, as specified in this model, as a function of location within geographic range.

\begin{tabular}{|c|c|c|}
\hline $\begin{array}{c}\text { Location within } \\
\text { geographic range }\end{array}$ & Mean clutch size & S.D. \\
\hline South & 7.00 & 2.5 \\
\hline Central & 6.25 & 2.0 \\
\hline Periphery & 5.00 & 1.5 \\
\hline
\end{tabular}




\section{Mortality rates}

VORTEX ${ }^{\circledR}$ models survivorship as age-specific mortality rates that remain constant once individuals reach maturity. Mortality rates reported for gopher tortoises in the literature are generally reported in relation to life stage rather than age of individuals. This model specified different mortality rates for hatchlings, yearlings, juveniles, subadults and adults. Because age at onset of maturity varies in this model between males and females and as a function of geographic range $\&$ habitat quality, the ages corresponding to those life stages vary among model scenarios. Table 4 lists the annual mortality rates this model specified for the different life stages Age-specific mortality schedules for males and females under different site-specific conditions can be determined by referring to the table of age at first reproduction (see Table 1).

Annual mortality rates and their variability are poorly known for gopher tortoises due to the scarcity of long-term mark-recapture studies in the literature. In addition, tortoises are difficult to accurately age once they reach reproductive maturity and most studies have focused on adults, further limiting the information on age-specific survivorship of gopher tortoises. Hatchling survivorship rates were based on radio-telemetry studies of hatchlings (Butler and Sowell 1996; Epperson and Heise 2003; Pike and Seigel 2006). J uvenile survivorship rates were based on a radio-telemetry study by Wilson (1991). Adult survivorship data are not available for naturally occurring populations, but are presumably at least as high as longterm survival rates reported for translocated populations by Ashton \& Burke (2007; 98.5 percent) and Tuberville et al. (2008 [in review]; 98 percent). In the absence of other data, long-term adult survivorship data from translocated populations were used.

Table 4. Stage-specific mortality rates as specified in our model.

\begin{tabular}{|l|l|c|}
\hline \multicolumn{1}{|c|}{ Life stage } & \multicolumn{1}{|c|}{ Ages } & Annual \% mortality \\
\hline Hatchling & age 0 to 1 & 96 \\
\hline Yearling & age 1 to 2 & 55 \\
\hline Juvenile & age 2 to 4 & 25 \\
\hline Subadult & age 4 to (age at maturity* - 1) & 3 \\
\hline Adults & age at maturity to 60 & 1.5 \\
\hline *See table of age at first reproduction, p 13. \\
\hline
\end{tabular}




\section{Mate monopolization (\% of males in breeding pool - 100\%)}

Although some males may be excluded from the breeding pool as a result of female choice or male-male competition, there are no data to quantify this parameter. Therefore, all males were assumed to be potential breeders. However, should behavioral or genetic data be available for specific populations, mate monopolization could be incorporated into the model. Data suggesting that mating opportunities are limiting for males under low density conditions could be incorporated into the model as a densitydependent effect (see “Density dependent reproduction," above, p 13).

\section{Initial population size}

Each combination of parameters was performed on simulated populations with initial sizes of 20,40,100,250, 500, 1000, and 2500 tortoises to capture the range of population sizes likely to occur on discrete management units. Selected population sizes are also based on categories used in Cox (1989), Miller et al. (2001), and Smith et al. (2006).

VORTEX ${ }^{\circledR}$ allows the user to specify an exact distribution or to have the software generate a stable age distribution. This model used the softwaregenerated stable age distribution for the initial population (see Appendix A, Table A2, p 46).

\section{Carrying capacity}

This model assumes that growth of "at-risk" tortoise populations is less likely to be limited by site carrying capacity than by other factors, such as habitat destruction, road mortality, and previous collection or predation by humans. This study attempted to incorporate the potential effects of habitat quality on population dynamics elsewhere in the model (e.g., reproduction, age at first reproduction) rather than in the carrying capacity module. Following Miller et al. (2001), carrying capacity (K) was set at 10 times the initial population size to reflect the assertion that most "at-risk" populations are not currently at carrying capacity. However, there is an option to predict future changes in $\mathrm{K}$ as a result of habitat management, which might be useful for modeling some scenarios. Although not considered in this model, carrying capacity of recipient sites should be determined prior to conducting any translocations to determine target population size and number of animals to be released. See Guyer et al. (in press) for guidance on estimating site carrying capacity for gopher tortoises. 


\section{Effects of Geographic Location, Habitat Quality, and Initial Gopher Tortoise Population Size}

Too few data are available to separate out the individual effects of geographic location and habitat quality on population rates. Therefore, a model was constructed to enable the simulation of several conditions as affecting population parameters simultaneously. Model results are discussed under the different combinations (i.e., scenarios) of geographic location, habitat quality, and initial population size.

\section{Deterministic population growth rate}

All model scenarios resulted in a population decline of 1- 3 percent per year and varied as a function of both habitat quality and location within the range (Figure 2). Populations in optimal habitat at the southern extent were potentially the most stable whereas populations at the periphery exhibited the greatest potential declines, particularly under marginal habitat conditions. From a management perspective, improving habitat conditions should affect population growth rates positively in all cases, and hence, the long-term viability of individual populations.

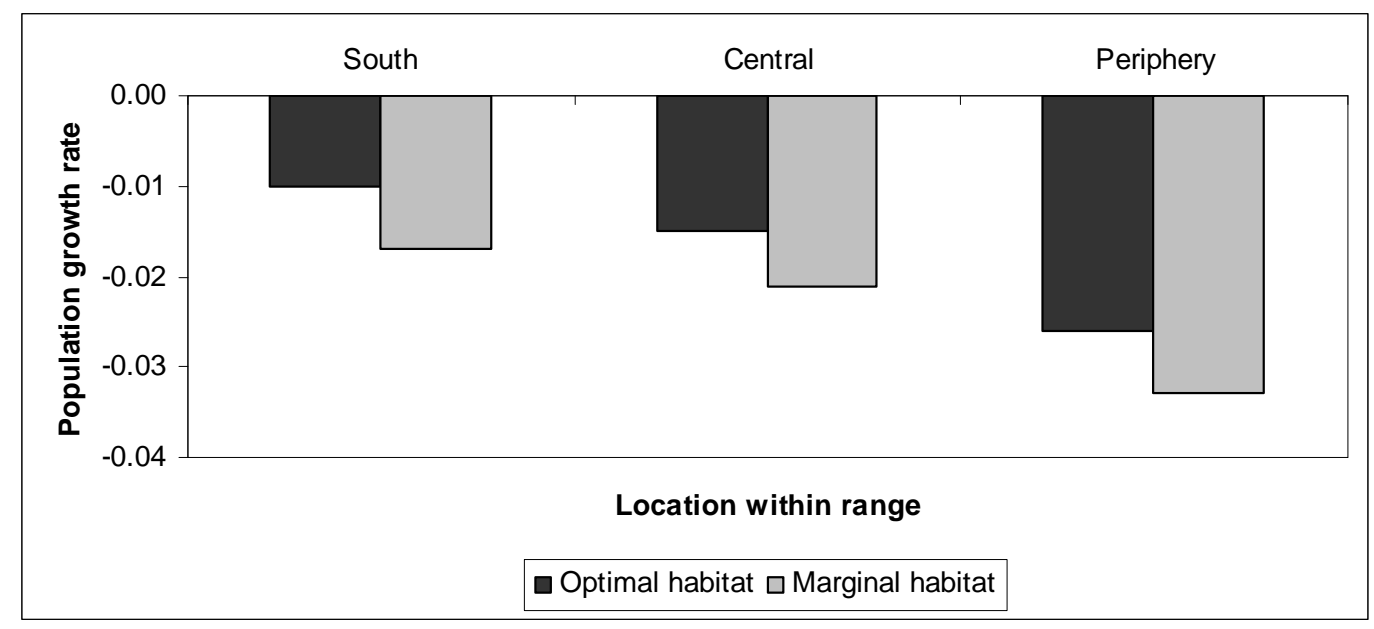

Figure 2. Deterministic population growth rate of simulated gopher tortoise populations in optimal (black bars) and marginal (gray bars) habitat conditions in different regions within the geographic range. 


\section{Probability of extinction}

Simulations were run for 200 years, the maximum duration possible within the VORTEX ${ }^{\circledR}$ program, to capture as many generation times for gopher tortoises as possible (Figure 3). However, because it is difficult to predict the magnitude of actual changes in extrinsic factors (e.g., climate, habitat fragmentation) and their effects on tortoise populations over such a long time, simulation results are also shown after 100 years (Figure 4).

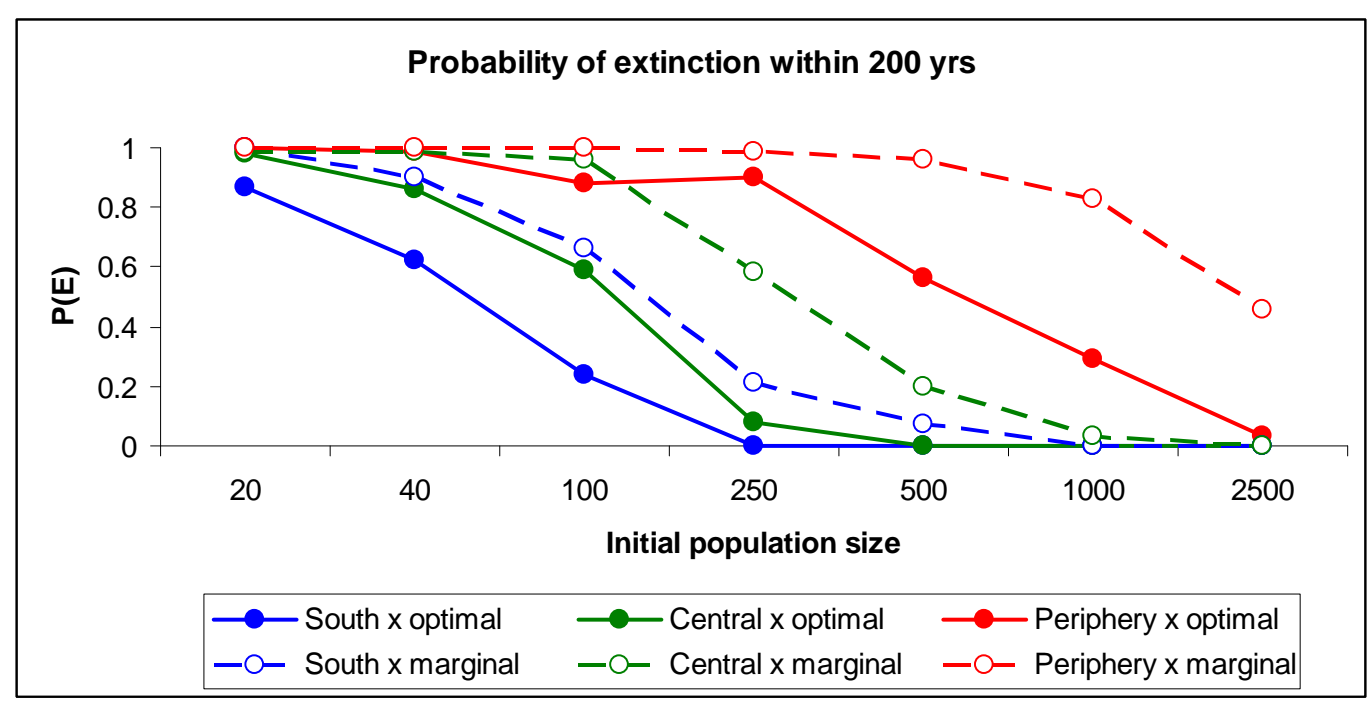

Figure 3. Probability of extinction within 200 years for simulated gopher tortoise populations of different initial population sizes based on the population's location within the range and habitat conditions. (Note: $x$-axis scale is not linear.)

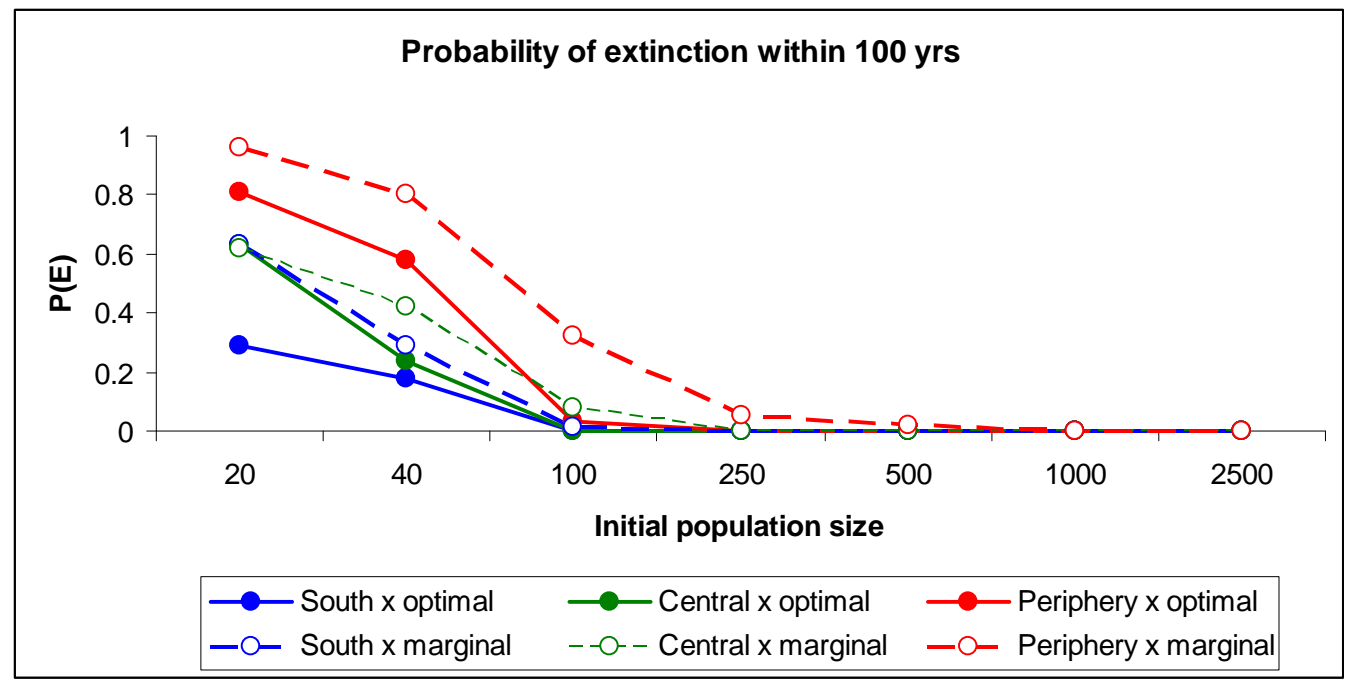

Figure 4. Probability of extinction within 100 years for simulated gopher tortoise populations of different initial population sizes based on the population's location within the range and habitat conditions. (Note: $x$-axis scale is not linear.) 
The probability of population extinction (defined here as when only one sex remains) increases with decreasing habitat quality and as a function of location within the range. Populations at the northern periphery are the most vulnerable, with populations of 500 tortoises in optimal habitat having 60 percent probability of extinction within 200 years (Figure 3). Under more favorable combinations of geographic location and habitat quality, populations of 250 tortoises are much less likely ( $0-20$ percent probability, except for central populations in marginal habitat) to become extinct within 200 years.

Over the first 100 years of simulation, populations of at least 100 tortoises are reasonably resilient to variation in habitat quality and location within the geographic range (Figure 4). Only populations at the periphery and in marginal habitat have a significant chance of extinction (30 percent) given that initial size; populations of 250 tortoises, however, have only a 5 percent chance of extinction under those same conditions.

\section{Population persistence}

Extinction under most scenario combinations of geographic range, habitat quality, and initial population size occurred (if at all) between 100- 200 years within the simulations (Figure 5, already shown in Figures 3 and 4). At any of the geographic locations, populations in optimal habitat persisted longer than populations of the same size in marginal habitat. Note that this may represent as few as 3- 4 generations for this species in which adults are very long-lived.

\section{Size of extant populations}

Under most scenarios, extant populations have fewer than 100 tortoises after 200 years, even when initial population sizes were as large as 1000 tortoises (Figure 6). Populations starting with 500 or more tortoises retain at least 100 tortoises at the end of the first 100 years, except at the periphery of the species' range (Figure 7).

\section{Summary of baseline model results}

Only initial populations of at least 250 tortoises were able to persist for 200 years. Within a 100-year time frame, initial populations of at least 100 animals were relatively robust, regardless of location within range and habitat quality (except under marginal habitat conditions at edge of range). 


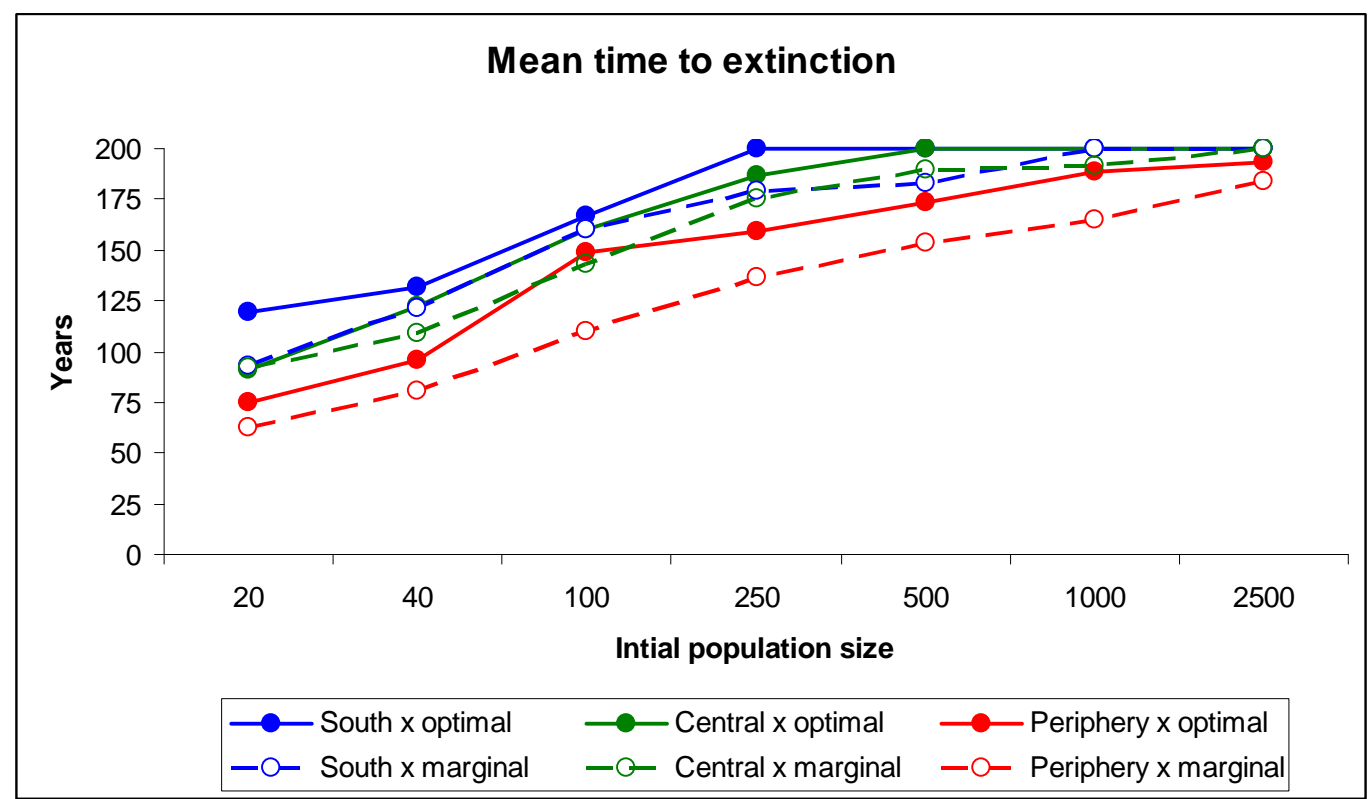

Figure 5. Population persistence (or mean time to extinction) for simulated gopher tortoise populations of different initial population sizes based on the population's location within the range and local habitat conditions. Graphs plateau at 200 years, the maximum simulation duration possible. (Note: $x$-axis scale is not linear.)

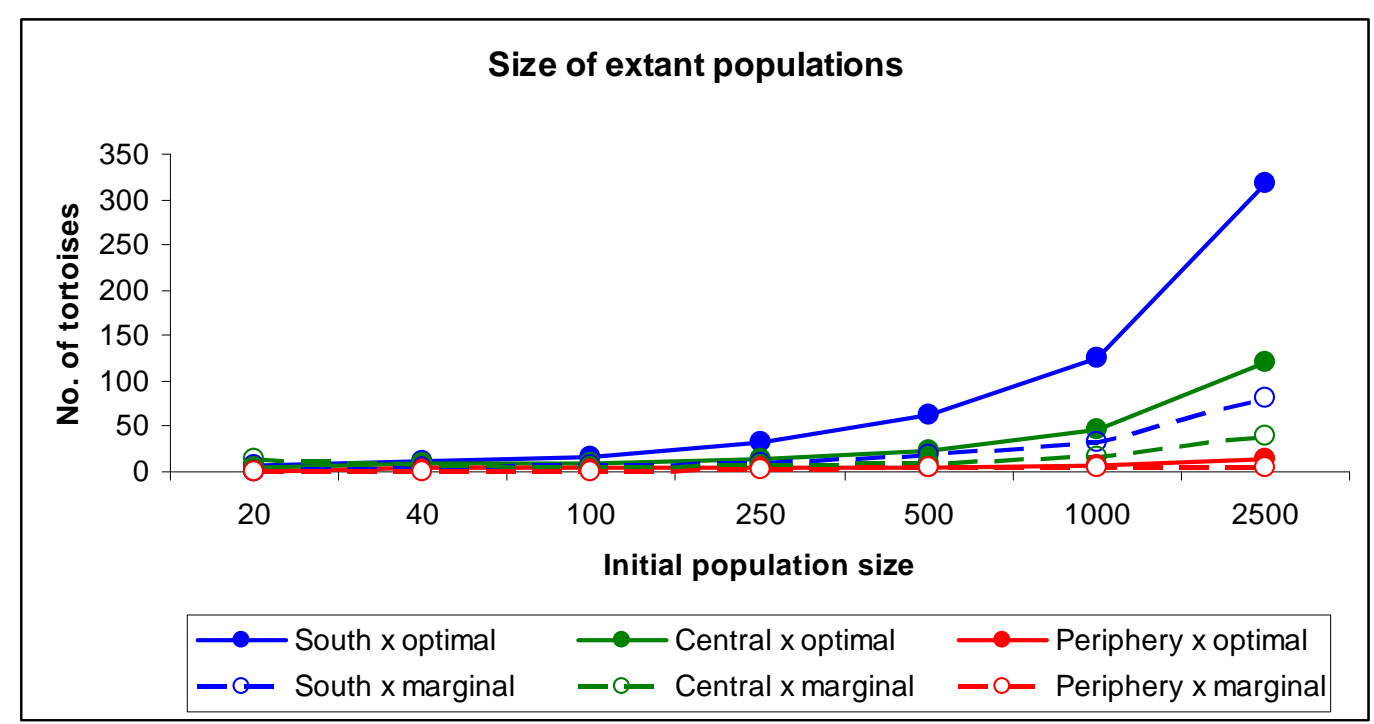

Figure 6. Size of populations extant after 200 years based on their initial population size, location with the geographic range (south, central, periphery) and habitat quality (optimal, marginal). (Note: $x$-axis scale is not linear.) 


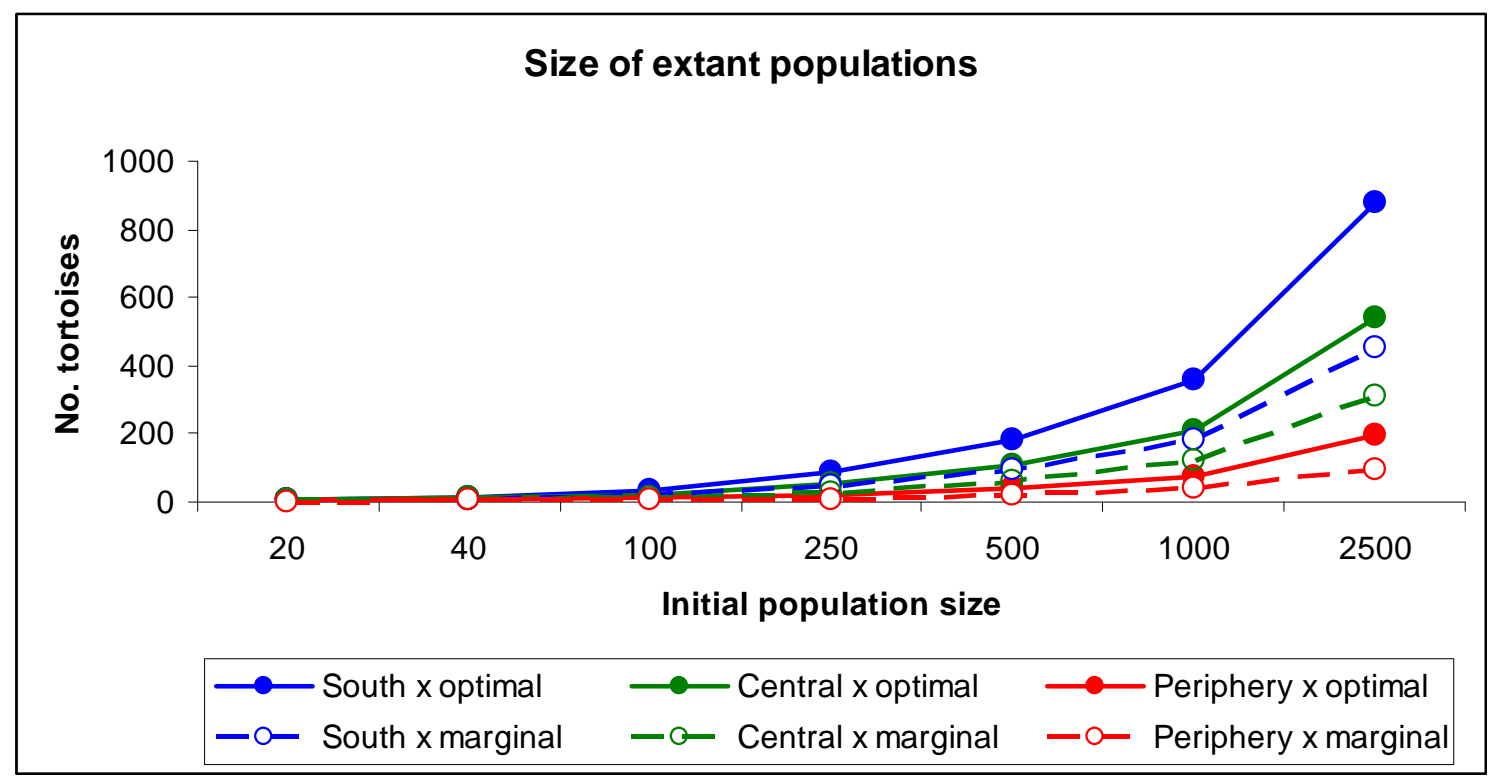

Figure 7. Size of populations extant after 100 years based on their initial population size, location with the geographic range (south, central, periphery) and habitat quality (optimal, marginal). (Note: $x$-axis scale is not linear.)

Populations at the periphery of the species' range were more vulnerable to extinction than populations in the central and south portion of the range, even under optimal habitat conditions and relatively large (>500 tortoises) initial population sizes. Not surprisingly, populations occurring in optimal habitat performed better than populations in marginal habitat. Due to longevity of individual animals, tortoise populations can persist for long periods of time in marginal habitat, making it difficult to detect subtle population declines (McCoy and Mushinsky 1992). If gopher tortoises exhibit low site fidelity due to poor habitat conditions (Aresco and Guyer 1999b; Guyer and Hermann 1997), then this model may underestimate population extinction in those habitats. However, these models demonstrate that the likelihood of long-term persistence of populations occurring in marginal habitat can be increased through effective habitat management.

Under all scenarios modeled, regardless of geographic location and habitat quality, gopher tortoise populations exhibited gradual declines. There are several possible explanations as to why these models predicted universal declines, including, but not limited to:

1. Some assumptions of the VORTEX ${ }^{\circledR}$ software program may make the program inappropriate for modeling the gopher tortoise's life history. There are two potentially important assumptions of the VORTEX ${ }^{\circledR}$ software program that may not be applicable to gopher tortoises. The first is that the species modeled has age-dependent vs. size-dependent survivorship. Al- 
though survivorship may in fact be more closely correlated with size or stage than with age, too few survivorship data are currently available to make this distinction meaningful in terms of model construction. In addition, size and age are highly correlated for any one site, although individual growth rates vary both regionally and locally (Mushinsky et al. 1994). This work attempted to capture the relationship between size, age, and stage by varying age at maturity as a function of geographic range and habitat quality. The second important assumption of the VORTEX ${ }^{\circledR}$ software is that survivorship and reproductive parameters do not increase with age after individuals reach maturity. Although this assumption may be violated in some turtle species, no data are available to determine whether these parameters change with age (independent of size) in adult gopher tortoises, or to model such age-related changes using other software programs.

2. The parameter estimates were derived from data collected on declining populations. If the parameter estimates in this model do adequately represent the population dynamics of currently extant gopher tortoise populations, then the species may be experiencing a range-wide decline even under the most favorable conditions. Results of demographic feasibility and sensitivity analyses (see following sections) should provide guidanœe on the relative likelihood that the declines exhibited in simulated populations are due to parameter uncertainty, or that they parallel declines experienced by real populations.

3. The life history of the species was not appropriately represented by the parameter values specified in the models. Although numerous studies have been conducted on gopher tortoises throughout their range, few published data from long-term population studies are available for estimating certain model parameters, particularly longevity and survivorship - both of which are identified as critical research needs for effective conservation of the species (Smith et al. 2006). Demographic sensitivity analysis (see the following section) can be used to identify the parameters that have the most influence on model outcome. These "sensitive" parameters can be come the focus of research and/ or management efforts, depending on the level of uncertainty associated with their estimates. Also, because the parameters used here were estimated from data collected from multiple populations, the combination of parameters used to construct the model may not reflect the population dynamics of any individual population. The demographic feasibility analysis should identify combinations of parameters that could produce a stable population. 


\section{Demographic feasibility analysis}

Because all the scenarios modeled in VORTEX ${ }^{\circledR}$ resulted in declining populations, it was desirable to evaluate whether the combination of demographic parameters currently available in the literature (and used in this model) represent a feasible demography for gopher tortoises. The term "feasible demography" is defined here (per Dunham and Overall 1994) as "any combination of average ... survivorship and fecundity values [that] allows long-term population persistence." Following methods by Dunham and Overall (1994) and Congdon et al. (1993, 1994), standard demographic analyses were conducted, manipulating only a single variable at a time, to identify the combination of values that produced the most stable population. The model is not intended to describe any single population of tortoises or any particular scenario modeled in VORTEX ${ }^{\circledR}$, but rather a "typical" or "average" sustainable tortoise population.

The following variables were fixed at constant values, based on literature specifically for gopher tortoises or from life history data from other turtle species, as indicated below:

- Annual fecundity $\left(\mathrm{m}_{\mathrm{x}}\right)$, the number of female eggs produced annually, based on the assumptions of an equal primary sex ratio, a mean clutch size of seven eggs (Landers et al. 1980; Mushinsky et al. 1994), and that 95 percent of adult females reproduce annually (Miller et al. 2001) and lay no more than one clutch annually.

- Adult survivorship ( $\mathrm{S}_{\mathrm{x} \text { Adult }}$ ), the proportion of adult females surviving each year, assuming consistently high survivorship (97 percent) among all adult age classes. High adult survivorship is supported by long-term mark-recapture studies of translocated gopher tortoise populations in Florida (Ashton and Burke 2007) and Georgia (Tuberville et al., 2008 [in review]). High adult survivorship has also been documented in long-term studies of other turtle species (Congdon et al. 1993, 1994, 2003; Frazer and Gibbons 1990; Gibbons 1987; Mitchell 1988).

- Nest survivorship ( $\mathrm{S}_{\mathrm{x} \text { Nest}}$ ), the proportion of embryos surviving the period between oviposition and emergence from nests. There are few data available for estimating survivorship of unprotected gopher tortoise nests; survivorship for protected nests varies from 40- 86 percent (Pike and Seigel 2006; Butler and Hull 1996; Epperson and Heise 2003; Smith 1995). Nest survivorship values (50 percent) were selected based on values reported for Chrysemys picta (Tinkle et al. 1981; Congdon et al. 2003), whose nesting ecology is well-described, and which, like the gopher tortoise, is a species that nests cryptically. 
The following variable, for which the fewest data are available, was manipulated:

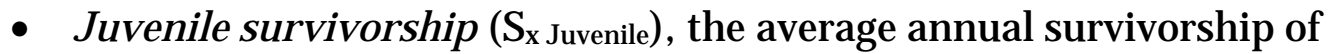
juvenile females between ages one and age at maturity. Based on comparison of results from studies of gopher tortoise hatchlings (Pike and Seigel 2006; Butler and Sowell 1996; Epperson and Heise 2003) and older juveniles (Wilson 1991; Tuberville et al., 2008 [in review]), survivorship of juveniles varies over the juvenile period, increasing with size and/ or age. For simplicity, however, it was assumed that annual survivorship was consistent throughout the juvenile period and lower than in adults. Because juvenile survivorship is poorly known in tortoises, different juvenile survivorship values were used to determine the value that resulted in the most stable population.

The life-table model calculates the following measures of population change for each combination of juvenile survivorship and the other (fixed) demographic variables:

- Reproductive rate $\left(\mathrm{R}_{0}\right)$, the mean number of female offspring produced per original female by the end of the cohort (i.e., death of the oldest female in the cohort; a value that indicates both average number of female offspring produced by a female over her lifespan, and the population multiplication factor that will indicate the size of the population in the next generation). Population sizes will decrease when $R_{0}<1.0$.

- Intrinsic rate of natural increase ( $r$ ), the change in population size per individual per unit time. Population sizes will decrease when $r<1.0$.

- Population doubling time ( $\mathrm{D}_{\text {Time }}$ ), the number of years required to double (positive value) or halve (negative value) the population size as a consequence of changes in survivorship.

Rather than focusing on the values of $R_{0}$ or $r$, population doubling-time was chosen as a more intuitive measure of population change. Table 5 lists the combination of demographic variables that resulted in the most stable population (i.e., the largest population doubling time).

When detailed demographic data are available for a stable population of interest, both the population modeling approach in VORTEX ${ }^{\circledR}$ and the feasible demography approach can be used to predict the population's response to specific demographic perturbations. However, when data are limited, the feasible demography approach can be used to develop estimates for poorly known demographic traits (e.g., juvenile survivorship) based on values of other demographic variables for which better estimates are available. 
Table 5. A summary of demographic values that produced the most stable population of gopher tortoises in the demographic analysis, and the resulting measures of population change.

\begin{tabular}{|l|l|}
\hline \multicolumn{2}{|c|}{ Reproduction } \\
\hline \multicolumn{2}{|c|}{ Survivorship (Ix) } \\
\hline Nest (age 0) & 0.3 \\
\hline Juvenile (ages 1-14) & 0.5000 \\
\hline Adult females (ages 15+) & 0.7475 \\
\hline \multicolumn{2}{|c|}{ Stable population parameters } \\
\hline Basic reproductive rate (Ro) & 1.9700 \\
\hline Intrinsic rate of population increase $(r)$ & $1.8820 \times 10-4$ \\
\hline Population doubling time (Dtime) & 3682.93 \\
\hline
\end{tabular}

In this model, it was estimated that annual juvenile survivorship must average approximately 75 percent over the juvenile period to produce a stable population - a value much higher than generally reported in the literature for the early juvenile years i.e., ages 0- 4 (Butler and Sowell 1996; Epperson and Heise 2003; Pike and Seigel 2006; Wilson 1991; Tuberville et al. 2008 [in review]). The feasible demography approach reveals that the combination of parameters used in VORTEX ${ }^{\circledR}$ are unlikely to produce a stable population under any of the scenarios modeled. It is therefore concluded that one or a combination of the following are true:

1. Short-term studies do not always allow adequate estimation of long-term demographic rates

2. J uvenile survivorship increases dramatically over the juvenile period

3. The demographic estimates reported in the literature are based on studies of declining populations.

\section{Demographic sensitivity analysis results}

Sensitivity analysis can be a useful tool for identifying the parameters that exert the strongest influence on model outcomes by varying individual parameters one at a time. If a high degree of uncertainty is associated with either the parameter estimates themselves, or with how they are used to construct the model, those parameters should become research or monitoring priorities so that better data can be obtained. If a parameter with strong influence on model outcome is already well understood, it may be an effective management target.

For the sensitivity analysis conducted here, a baseline model was used for a population of 100 tortoises located in the central geographic range and 
occupying optimal habitat. This baseline model was chosen to simulate conditions likely to be encountered on public conservation lands within the core of the species' range. Based on the results presented in the previous section, initial population sizes of 100 were the smallest populations resilient to variation in geographic locations and habitat quality and were therefore considered "viable" over the long term (cf. Figure 4). In addition, many public conservation lands are likely to be large enough to support a population of 100 tortoises if habitat is managed appropriately.

For each parameter that was manipulated individually (see Appendix A, Table A3, $p$ 47), the corresponding changes in deterministic population growth rate, probability of extinction within 200 years, and mean time to extinction are discussed in the following sections.

\section{Maximum age of reproduction (longevity)}

Adult gopher tortoises presumably continue to reproduce throughout their lives, so maximum age at reproduction is essentially equivalent to longevity for the purposes of this model. However, maximum or even average longevity of this species is not known with any certainty, but was set at 60 years in this baseline model. For every 10 yr change in expected longevity, there was a corresponding difference of $10-30$ percent in the probability of extinction and a 10-40 year change in estimated population persistence (Figure 8). Population growth rates were still slightly negative when longevity was set at 100 years, but populations were not likely to decline to extinction within 200 years.

\section{Clutch size}

Clutch sizes are fairly well-documented throughout the species' range (e.g., Butler and Hull 1996 [north FL]; Diemer and Moore 1994 [north-central FL]; Epperson and Heise 2003 [MS]; Landers et al. 1980; Mushinksy et al. 1994 [central FL]; Rostal and J ones 2002 [southeast GA]; Smith 1995 [FL]; Smith et al. 1997 [LA, MS]; Wright 1982 [SC]). The mean clutch sizes used in this model scenarios seem to be reasonable estimates, suggesting that uncertainty in this parameter is unlikely to play an important role in model predictions. However, management actions have the potential to influence mean clutch size of a population. Habitat management (or an absence thereof) can influence growth rates (Aresco and Guyer 1999a) and, therefore, the age at which females are recruited into the breeding pool and the mean body sizes of those females. 

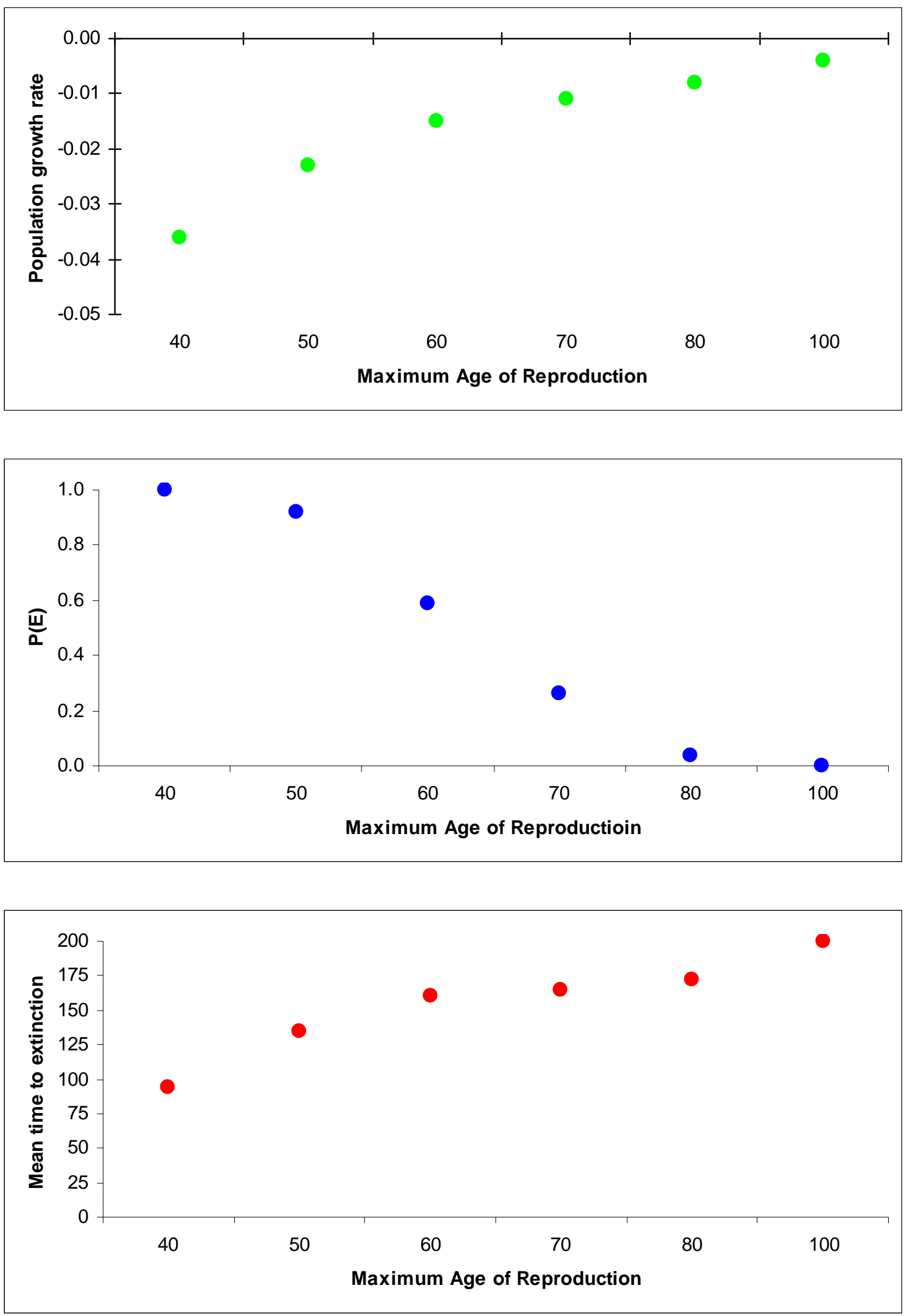

Figure 8. Population growth rates (top), probability of extinction within 200 years (center), and mean time to extinction (bottom) as a function of maximum age of reproduction. 
In addition, females living in habitats with abundant, high quality forage are likely to have more resources to devote to reproduction than females of the same size living in poor quality habitats. Therefore, clutch size was included in this demographic sensitivity analysis.

An increase or decrease in mean population clutch size by two eggs resulted in no more than a 1 percent corresponding change in the population growth rate (Figure 9). Although this clutch size increase was not sufficient to result in a stable population growth rate (i.e., equal to 0 ), it did result in a predicted 40 percent decrease in the probability of population extinction within 200 years and a 25 year increase in population persistence time. It is not known how large a change in mean clutch size could be produced as a result of habitat manipulations.

\section{Sex ratio}

The effect of sex ratio bias on model outcome was examined by varying the proportion of females in the initial population from 40-60 percent. Although sex ratio among clutches may vary as a result of different thermal conditions among nests, the overall sex ratio of hatchlings within a population is presumably close to 1:1. And while sex-biased mortality has been documented in adult aquatic turtles (e.g., nesting females are more susceptible to road mortality than males in some species), no published data have demonstrated that mortality rates vary between male and female gopher tortoises after hatching. An analysis of long-term mark-recapture data from a translocated population on a privately owned island did not detect any differences in mortality rates between adult males and adult females (Tuberville et al. 2008 [in review]; Tuberville 2008). Theoretically, dramatic climate change could eventually influence nest temperatures and sex ratios, with higher nest temperatures producing a higher proportion of females. Nest temperatures could also be manipulated through artificial incubation in the lab, although this is a manually intensive endeavor. Adult sex ratios could be manipulated in translocated populations during the selection of release animals.

For every 5 percent increase in the proportion of adult females comprising the population, population growth rate increased by $<0.3$ percent, probability of extinction within 200 years decreased by $10-20$ percent, and mean time to extinction increased by 5- 10 years (Figure 10). 

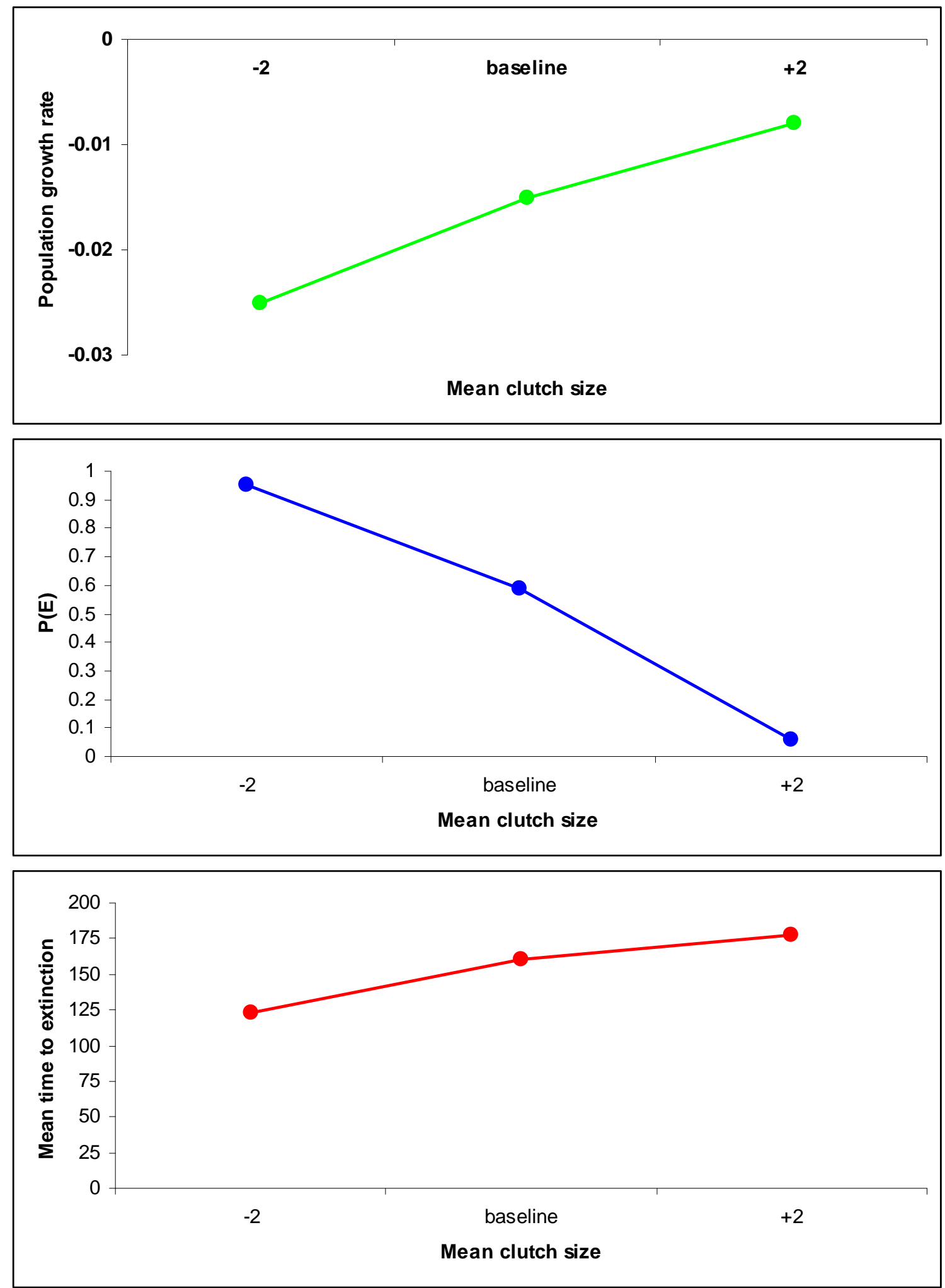

Figure 9. Population growth rates (top), probability of extinction within 200 years (center), and mean time to extinction (bottom) as a function of mean clutch size. (The baseline clutch size is 6.75 eggs.) 

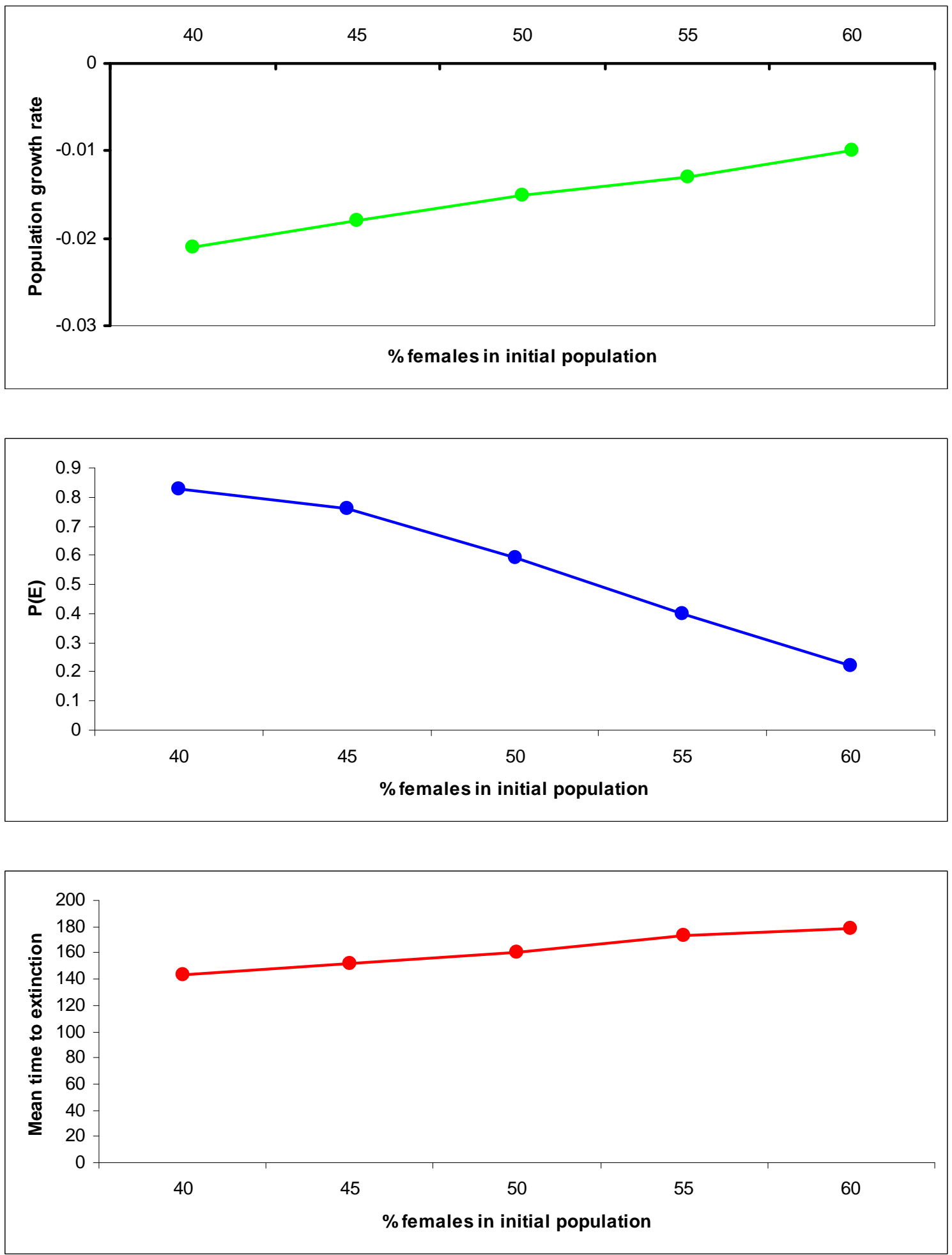

Figure 10. Population growth rates (top), probability of extinction within 200 years (center), and mean time to extinction (bottom) as a function of sex ratio (\% females in initial population). 
Given the relative resilience of the model to variation in sex ratio, the lack of evidence that sex ratios are likely to be skewed in natural populations of gopher tortoises, and the difficulty in artificially manipulating sex ratios, sex ratios are not an efficient management target.

\section{Proportion of females breeding}

The proportion of female gopher tortoises breeding in a population is not well-documented in the literature. Although short-term studies have reported the percent of females encountered with eggs, it is difficult to determine to what extent these values reflect actual reproduction rates in the population versus the methodology of the study. (In other words, it was not known if females captured without eggs had already nested or would nest later in the season.) In addition, few data are available on annual variation and site-specific variation in this parameter. In addition to the high degree of uncertainty regarding the range of values for this parameter in natural populations, the proportion of females breeding is also not likely to be specifically manipulated, although the proportion may vary as a function of habitat quality.

The effect of variation in proportion of females breeding on population dynamics was examined by varying the percentage of females breeding from 80- 100 percent, which encompasses the primary values reported in the literature (Rostal and J ones 2002; Diemer and Moore 1994) and the range in values considered likely to occur in natural populations. For every 10 percent increase in the proportion of females breeding, the population growth rate increased by 0.3 percent, probability of extinction within 200 years decreased by approximately 15 percent, and mean time to extinction increased by $<5$ years (Figure 11). This parameter, therefore, is not an efficient management target, and although poorly understood for natural populations, does not appear to be a significant driver of model outcome.

\section{Comparative survivorship of immature stages}

Adult mortality is widely recognized as being the primary force driving population dynamics for most turtle species (Congdon et al. 1993, 1994). However, because models in this study already assume a naturally low annual adult mortality rate ( 1.5 percent) and still predict population declines under all scenarios simulated, the focus here is on the immature classes. Mortality rates are uncertain for these age classes, and because mortality rates are likely to be higher for these age classes than for adults, decreasing these mortality rates could prove to be effective management goal. 

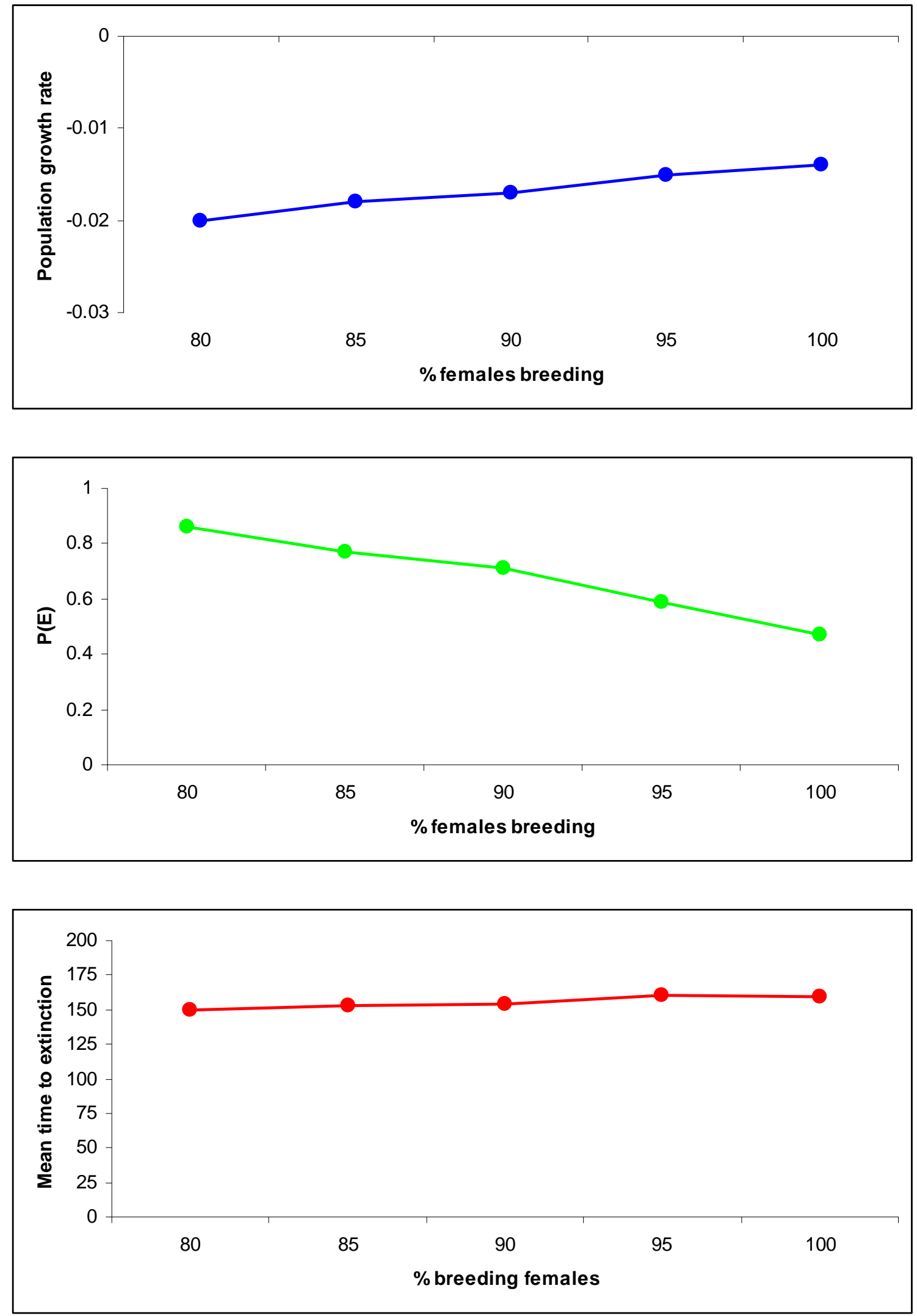

Figure 11. Population growth rates (top), probability of extinction within 200 years (center), and mean time to extinction (bottom) as a function of proportion of females breeding. 
In the baseline model, the population growth rate was - 1.5 percent, resulting in a population with an initial size of 100 tortoises having a 60 percent probability of extinction within 200 years and a mean time to extinction of 160 years. The model was more responsive to changes in juvenile mortality rates than to yearling mortality rates, probably due to the longer duration of the juvenile stage (age 2-4) compared to the yearling stage. The model was most responsive to changes in hatchling mortality. A 5 percent decrease (from 96 percent in the baseline model to 91 percent) in hatchling mortality was sufficient to shift the population growth rate from slowly declining ( -1.5 percent) to slowly increasing $(+1.1$ percent) and to eliminate the probability of extinction within the next 200 years (Figure 12). The hatchling mortality rate used in the baseline model represents a severe bottleneck to recruitment and long-term population viability, highlighting the need for a better understanding of hatchling mortality rates in natural populations.

Hatchling mortality rates have been reported in three published radiotelemetry studies from Florida and Mississippi (Butler and Sowell 1996; Epperson and Heise 2003; Pike and Seigel 2006). The naturally high mortality rates combined with the intensive nature of radio-telemetry studies make it difficult, however, to obtain sample sizes large enough to estimate hatchling survivorship and its variability accurately (Pike and Seigel 2006). The changes in hatchling mortality rates necessary to produce significant changes in population dynamics (Figure 13) are small enough to fall within the range of both measurement error/ parameter uncertainty and natural variation among years and/ or among sites. Further research on hatchling mortality is warranted, and effective management of individual populations may require some basic understanding of site-specific hatchling mortality rates. At the very least, should managers suspect that hatchling survivorship is low or non-existent, then management actions to increase hatchling survivorship - such as predator control, habitat enhancement, or perhaps even head-starting - should be considered. Prehatching clutch losses may potentially be minimized as well through nest protection measures. 

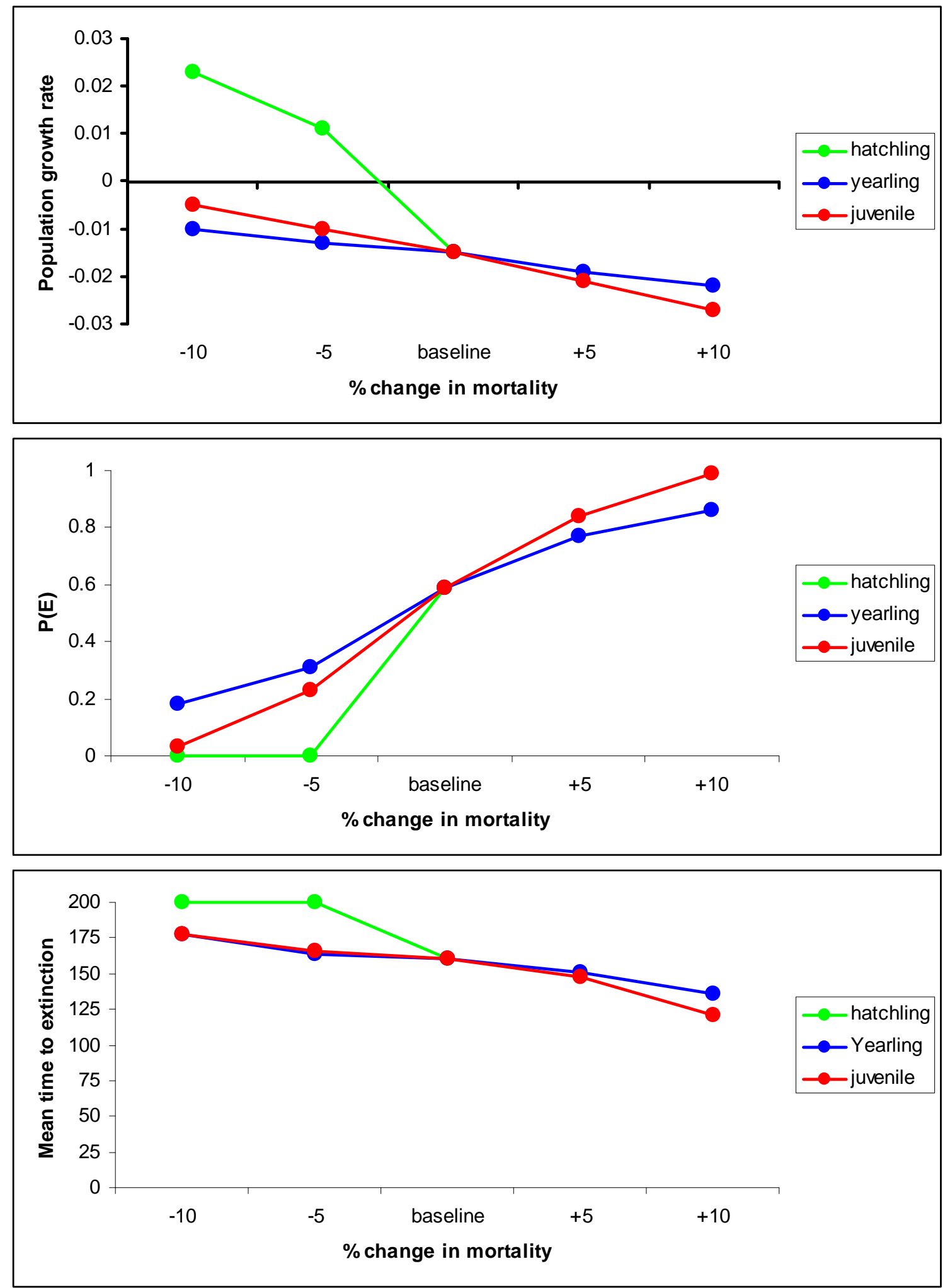

Figure 12. Population growth rates (top), probability of extinction within 200 years (center), and mean time to extinction (bottom) as a function of variation in hatchling, yearling and juvenile mortality rates from baseline model. 

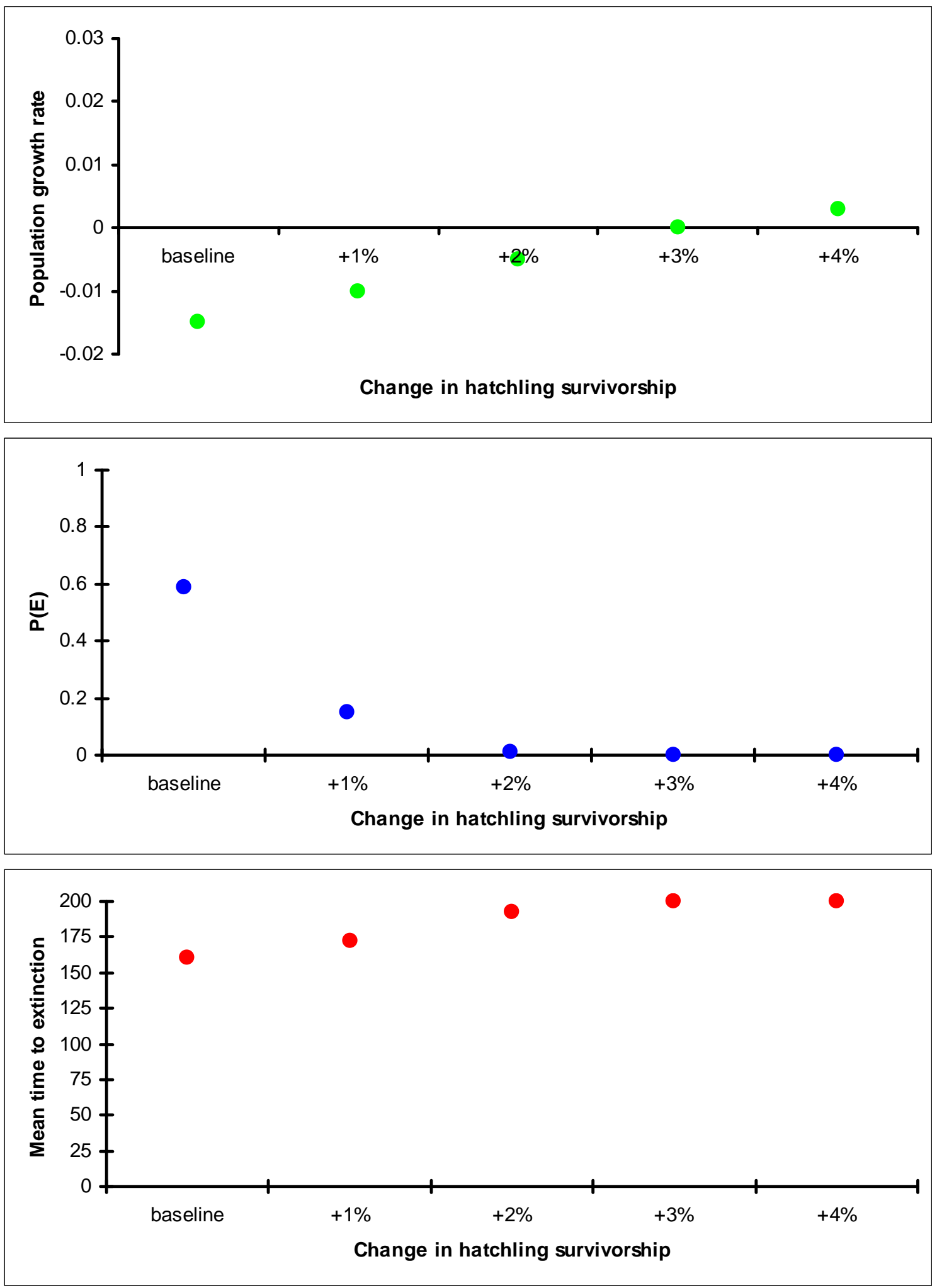

Figure 13. Population growth rates (top), probability of extinction within 200 years (center), and mean time to extinction (bottom) as a function of small variation in hatchling mortality rates from baseline model (baseline survivorship $=4 \%$ ). 


\section{Conclusions and Recommendations}

This study constructed baseline models for gopher tortoise populations using VORTEX ${ }^{\circledR}$ population viability analysis software from demographic values currently available in the literature. All baseline model scenarios resulted in declining populations, though populations of at least 100 animals were unlikely to experience extinction over the first 100 years of the simulations and populations of at least 250 animals were modeled as persisting for 200 years. The most likely causes for the predicted decline include: (1) demographic values reported in the literature are based on studies of declining populations, and (2) sufficient data for estimating some demographic parameters are currently lacking.

Gopher tortoises are currently recognized as threatened or endangered by every state in which they occur, are Federally listed in the western portion of their range (USFWS 1987), and proposed for listing in the remainder of their distribution (Save Our Big Scrub et al., 2006). Habitat destruction, fragmentation, and degradation as a result of development, intensive silviculture, and fire suppression have undoubtedly reduced both the area and quality of suitable habitat throughout their range (Aresco and Guyer 1999b; Hermann et al. 2002; J ones and Dorr 2004). However, few longterm mark-recapture data sets are available for documenting trends of individual populations.

Based on indirect survey methods (i.e., surveys of burrows rather than direct observation of tortoises), McCoy et al. (2006) concluded that gopher tortoise populations experienced declines over a 10-year period at eight of 10 protected sites in Florida. A burrow survey conducted at a state preserve in South Carolina managed specifically for gopher tortoises estimated that over a 20-year period, the population experienced an annual decline of 2.33 percent (Tuberville and Dorcas 2001), which is very similar to the 2.6 percent annual decline predicted by this model for a population in optimal habitat at the periphery of the range. So, while there is some evidence for localized and even regional declines of gopher tortoises, it remains unclear the extent to which the declines predicted by these models were a result of demographic estimates obtained from declining populations. 
Regardless, this demographic feasibility analysis indicated that the combination of parameters used in this baseline model was unlikely to result in a stable population. Specifically, juvenile survivorship rates would need to be much higher than those reported in the literature. Demographic sensitivity analysis demonstrated that these models were very sensitive to changes in survivorship of the immature stages. More comprehensive data on juvenile survivorship and its variability are essential to developing more realistic population models and to effectively manage tortoise populations. This is one aspect in which several possible types of management intervention may be practiced. "Head-starting," or fostering of hatchlings into their second or third year (or longer) has been practiced informally in many locations. It is not clear that head-starting has had a measurable long term effect on population viability, although the time frame for detecting an effect likely exceeds duration of current monitoring efforts. Management of predators, especially subsidized predators such as raccoons and skunks, may also decrease loss of both eggs and hatchlings.

Populations that are not likely to be viable will require some level of management intervention, including habitat and/ or population manipulations. In-situ protection and management are preferred, where possible. These models suggest that improved habitat conditions can improve long-term population viability and that populations at the northern periphery are more vulnerable to poor habitat conditions. When habitat management alone is unlikely to ensure population viability, manipulations of the population itself-such as head-starting, translocation, or augmentation-may be necessary.

Note, however, that the models and interpretations presented here are limited by the current understanding of gopher tortoise life history. As more comprehensive data become available, these models can be refined to more accurately depict tortoise life history and provide helpful guidance for future management of individual tortoise populations. 


\section{References}

Aresco, M. J., and C. Guyer. 1999a. Growth of the tortoise Gopherus polyphemus in slash pine plantations of southcentral Alabama. Herpetologica 55:499-506.

Aresco, M. J ., and C. Guyer. 1999b. Burrow abandonment by gopher tortoises in slash pine plantations of the Conecuh National Forest. J ournal of Wildlife Management 63:26- 35.

Ashton, K. G., and R. L. Burke. 2007. Long-term retention of a relocated population of gopher tortoises. J ournal of Wildlife Management 71:783- 787.

Boglioli, M. D., C. Guyer, and W. K. Michener. 2003. Mating opportunities of female gopher tortoises, Gopherus polyphemus, in relation to spatial isolation of females and their burrows. Copeia 2003:846-850.

Burke, R. L., M. E. Ewert, J . B. McLemore, and D. R. J ackson. 1996. Temperaturedependent sex determination and hatching success in the gopher tortoise (Gopherus polyphemus). Chelonian Conservation and Biology 2:86- 88.

Butler, J . A., and T. W. Hull. 1996. Reproduction of the tortoise, Gopherus polyphemus, in northeastern Florida. J ournal of Herpetology 30:14- 18.

Butler, J . A., and S. Sowell. 1996. Survivorship and predation of hatchling and yearling gopher tortoises, Gopherus polyphemus, J ournal of Herpetology 30:455- 458.

Congdon, J . D., A. E. Dunham, R. C. van Loben Sels. 1993. Delayed sexual maturity and demographics of Blanding's turtles (Emydoidea blandingii): Implications for conservation and management of long-lived organisms. Conservation Biology 7:826- 833 .

Congdon, J . D., A. E. Dunham, and R. C. van Loben Sels. 1994. Demographics of common snapping turtles (Chelydra serpentina): Implications for conservation and management of long-lived organisms.

Congdon, J . D., R. D. Nagle, O. M. Kinney, R. C. van Loben Sels, T. Quinter, and D. W. Tinkle. 2003. Testing hypotheses of aging in long-lived painted turtles (Chrysemys picta). Experimental Gerontology 38:765- 772.

Cox, J . 1989. Survival characteristics of small tortoise populations and their possible influence on relocation efforts. pp 7- 14 in J . E. Diemer, D. R. J ackson, J . L. Landers, J.N. Layne and D. A. Wood, eds. Gopher tortoise relocation symposium proceedings. Florida Game and Fresh Water Fish Commission Nongame Wildlife Technical Report \#5, Tallahassee, FL.

Cox, J ., D. Inkley, and R. Kautz. 1987. Section 2: Estimating population viability, minimum area requirements, and habitat quality for small gopher tortoise populations. pp 23- 35 in Ecology and habitat protection needs of gopher tortoise (Gopherus polyphemus) populations on lands slated for development in Florida. Florida Fish and Fresh Water Fish Commission Nongame Wildlife Program Technical Report No. 4. Tallahassee, FL. 
Demuth, J . P. 2001. The effects of constant and fluctuation incubation temperatures on sex determination, growth, and performance in the tortoise Gopherus polyphemus. Canadian J ournal of Zoology 79:1609- 1620.

Diemer, J . E., and C. T. Moore. 1994. Reproduction of gopher tortoises in north-central Florida. In: R. B. Bury, and D. J . Germano, eds. Biology of North American Tortoises. Fish and Wildlife Resarch 13:129- 137.

Doak, D., P. Kareiva, and B. Kleptatka. 1994. Modeling population viability for the desert tortoise in the western Mojave desert. Ecological Applications 4:446- 460.

Dunham, A. E., and K. L. Overall. 1994. Population responses to environmental change: Life history variation, individual-based models, and the population dynamics of short-lived organisms. American Zoologist 34:382- 396.

Epperson, D. M., and C. D. Heise. 2003. Nesting and hatchling ecology of gopher tortoises (Gopherus polyphemus) in southern Mississippi. J ournal of Herpetology 37:315- 324.

Eubanks, J. O., W. K. Michener, and C. Guyer. 2003. Patterns of movement and burrow use in a population of gopher tortoises (Gopherus polyphemus). Herpetologica 59:311- 321.

Frazer, N., and J. W. Gibbons 1990. Life tables of a slider population. Pages 183- 200 in J. W. Gibbons, ed. Life history and ecology of the slider turtle. Washington DC: Smithsonian Institution Press.

Gibbons, J . W. 1987. Why do turtles live so long? BioScience 37:262- 269.

Guyer, C., R. Birkhead, and H. Balbach. 2006. Effects of tracked-vehicle training activity on gopher tortoise (Gopherus polyphemus) behavior at Fort Benning, GA. ERDC/CERL TR-06-10/ADA467989, Champaign, IL: Engineer Research and Development Center Construction Engineering Research Laboratory (CERLERDC).

Guyer, C., and S. M. Hermann. Patterns of size and longevity of gopher tortoise (Gopherus polyphemus) burrows: Implications for the longleaf pine forest. Chelonian Conservation and Biology 2:507- 513.

Guyer, C., V. M. J ohnson, and S. M. Hermann. In press. What values for estimates of carrying capacity should be used for conservation of gopher tortoises? A report to CERL-ERDC.

Heppell, S. S. 1998. Application of life-history theory and population model analysis to turtle conservation. Copeia 1998:367- 375.

Heppell, S. S., D. T. Crouse, and L. B. Crowder. 2005. A population model to estimate recovery time, population size, and management impacts on Kemp's Ridley sea turtles. Cehlonian Conservation and Biology 4:767- 773.

Heppell, S. S., L. B. Crowder, and D. T. Crouse. 1996. Models to evaluate headstarting as a management tool for long-lived turtles. Ecological Applications 6:556- 565. 
Hermann, S. M., C. Guyer, J . H. Waddle, and M. G. Nelms. 2002. Sampling on private property to evaluate population status and effects of land use practices on the gopher tortoise, Gopherus polyphemus. Biological Conservation 108:289- 298.

Horne, B. D., R. J . Brauman, M. J . C. Moore, and R. A. Seigel. 2003. Reproductive and nesting ecology of the yellow-blotched map turtle, Graptemys flavimaculata: Implications for conservation and management. Copeia 2003:729- 738.

Iverson, J . B. 1980. The reproductive biology of Gopherus polyphemus (Chelonia, Testudinidae). American Midland Naturalist 103:353- 359.

J ohnson, V. M., C. Guyer, and M. D. Boglioli. 2007. Phenology of attempted matings in gopher tortoises. Copeia 2007:490-495.

J ones, J . C., and B. Dorr. 2004. Habitat associations of gopher tortoise burrows on industrial timberlands. Wildlife Society Bulletin 32:456- 464.

Lacy, R. C., M. Borbat, and J . P. Pollack. 2005. VORTEX: A stochastic simulation of the extinction process. Version 9.50. Brookfield, IL: Chicago Zoological Society.

Landers, J . L., J . A. Garner, and W. A. McRae. 1980. Reproduction of gopher tortoises (Gopherus polyphemus) in southwestern Georgia. Herpetologica 36:353- 361.

Landers, J . L., W. A. McRae, and J . A. Garner. 1982. Growth and maturity of the gopher tortoise in southwestern Georgia. Bulletin of the Florida State Museum, Biological Sciences 27:81- 110.

MacDonald, L. A. 1996. Reintroduction of gopher tortoises (Gopherus polyphemus) to reclaimed phosphate land. Bartow (FL): Florida Institute of Phosphate Research. Report No. 03-105-126, 56 pp.

MoCoy, E. D., and H. R. Mushinsky. 1992. Studying a species in decline: Changes in populations of the gopher tortoise on Federal lands in Florida. Florida Scientist 55:116- 125.

McCoy, E. D., H. R. Mushinsky, and J . Lindzey. 2006. Declines of the gopher tortoise on protected lands. Biological Conservation 128:120- 127.

Miller, P. S., and participants. 2001. Preliminary population viability assessment for the gopher tortoise (Gopherus polyphemus) in Florida. Gopher Tortoise Population Viability Workshop, Tallahassee, FL, 11- 12 September 2001. 45 pp.

Miller, P. S., and R. C. Lacy. 2005. VORTEX: A stochastic simulation of the extinction process. Version 9.50 user's manual. Apple Valley, MN: Conservation Breeding Specialist Group (SSC/IUCN).

Mitchell, J . C. 1988. Population ecology and life histories of the freshwater turtles Chrysemys picta and Sternotherus odoratus in an urban lake. Herpetological Monographs 2:40-61.

Moon, J . C., E. D. MoCoy, H. R. Mushinsky, and S. A. Karl. 2006. Multiple paternity and breeding system in the gopher tortoise, Gopherus polyphemus. J ournal of Heredity 97:150- 157. 
Mushinsky, H. R., D. S. Wilson, ad E. D. McCoy. 1994. Growth and sexual dimorphism of Gopherus polyphemus in central Florida. Herpetologica 50:119- 128.

Pedrona, M., L. L. Smith, J . Clobert, M. Massot, and F. Sarrazin. 2004. Wild-captive metapopulation viability analysis. Biological Conservation 119:463- 473.

Pike, D. A., and R. A. Seigel. 2006. Variation in hatchling tortoise survivorship at three geographic localities. Herpetological 62:125- 131.

Rivera, A. C., and C. A. Fernandez. 2004. A management plan for the European pond turtle (Emys orbicularis) populations of the Louro river basin (northwest Spain). Biologia 59:161- 171.

Rostal, D. C., and D. N. J ones, Jr. 2002. Population biology of the gopher tortoise (Gopherus polyphemus) in southeast Georgia. Chelonian Conservation and Biology 4:479- 487.

Save Our Big Scrub, Inc. and Wild South. 2006. Petition to list the eastern population of the gopher tortoise as a threatened species before the Secretary of the U.S. Interior and the Director of the U.S. Fish and Wildlife Service (USFWS), received 20 J anuary 2006.

Seigel, R. A., and C. K. Dodd, J r. 2000. Manipulation of turtle populations for conservation: Halfway technologies or viable options? pp 218-238 in M. W. Klemens, (ed.), Turtle Conservation. Smithsonian Press, Washington DC.

Smith, K. R., J . A. Hurley, and R. A. Seigel. 1997. Reproductive biology and demography of gopher tortoises (Gopherus polyphemus) from the western portion of their range. Chelonian Conservation and Biology 2:596-600.

Smith, L. L. 1995. Nesting ecology, female home range and activity, and population sizeclass structure of the gopher tortoise, Gopherus polyphemus, on the Katherine Ordway Preserve, Putnam County, Florida. Bulletin of the Florida Museum of Natural History 37:97- 126.

Smith, L. L., T. D. Tuberville, and R. A. Seigel. 2006. Workshop on the ecology, status, and management of the gopher tortoise (Gopherus polyphemus), J oseph W. J ones Ecological Research Center, Newton, GA, 16- 17J anuary 2003: Final results and recommendations. Chelonian Conservation and Biology 5: 326- 330.

Tinkle, D. W., J . D. Congdon, and P. C. Rosen. 1981. Nesting frequency and success: Implications for the demography of painted turtles. Ecology 62:1426- 1432.

Tuberville, T. D. 2008. Evaluating the success of translocations for turtle conservation: A case study based on behavioral and demographic responses of the gopher tortoise, Gopherus polyphemus. Ph.D. dissertation, University of Georgia, Athens, GA.

Tuberville, T. D., E. E. Clark, K. A. Buhlmann, and J. W. Gibbons. 2005. Translocation as a conservation tool: Site fidelity and movements of repatriated gopher tortoises (Gopherus polyphemus). Animal Conservation 8:349- 358.

Tuberville, T. D., and M. E. Dorcas. 2001. Winter survey of a gopher tortoise population in South Carolina. Chelonian Conservation and Biology 4:182- 186. 
Tuberville, T. D., T. M. Norton, B. D. Todd, and J . S. Spratt. 2008 (in review). Long-term apparent survival of translocated gopher tortoises: A comparison of newly released and previously established animals. Submitted to Biological Conservation 29 J anuary 2008.

Wilson, D. S. 1991. Estimates of survival for juvenile gopher tortoises, Gopherus polyphemus. J ournal of Herpetology 25:376- 379.

Wright, J . S. 1982. Distribution and population biology of the gopher tortoise, Gopherus polyphemus, in South Carolina. M.S. Thesis, Clemson University, SC. 


\section{Acronyms and Abbreviations}

$\begin{array}{ll}\text { Acronym } & \text { Spellout } \\ \text { ACSIM } & \text { Assistant Chief of Staff for Installation Management } \\ \text { CERL } & \text { Construction Engineering Research Laboratory } \\ \text { ERDC } & \text { Engineer Research and Development Center } \\ \text { IUCN } & \text { International Union for Conservation of Nature and Natural Resources } \\ \text { LA } & \text { load allocation } \\ \text { PVA } & \text { Population Viability Analysis } \\ \text { SREL } & \text { Savannah River Ecology Laboratory } \\ \text { SSC } & \text { Species Survival Commission } \\ \text { TES } & \text { threatened and endangered species } \\ \text { TR } & \text { Technical Report } \\ \text { URL } & \text { Universal Resource Locator } \\ \text { URTD } & \text { Upper Respiratory Tract Disease } \\ \text { USFWS } & \text { U.S. Fish and Wildlife Service } \\ \text { WWW } & \text { World Wide Web }\end{array}$




\section{Appendix A: Parameter estimates used in the VORTEX ${ }^{\circledR}$ baseline model for gopher tortoise populations}

Important model assumptions include:

1. Sex ratio is $1: 1$

2. Mortality is equal between males and females

3. Mortality is stage-based rather than age-based

4. Fecundity does not increase with age

5. Dispersal is equivalent to death of the individual. 
Table A1. Parameter estimates used in the VORTEX ${ }^{\circledR}$ baseline model for gopher tortoise populations.

\begin{tabular}{|c|c|c|}
\hline Model input parameter & $\begin{array}{l}\text { Parameter } \\
\text { estimate }\end{array}$ & Justification \\
\hline Breeding system & polygynous & \\
\hline Age at first reproduction (F/M) & & \multirow{7}{*}{$\begin{array}{l}\text { Site-specific factors (such as location within } \\
\text { geographic range and habitat quality) will have } \\
\text { systematic effects on tortoise growth and, as a } \\
\text { result, age at maturity. Although length of the } \\
\text { activity season (and opportunity for growth) } \\
\text { varies with latitude, there is also evidence } \\
\text { growth rates among tortoises can vary signifi- } \\
\text { cantly among local populations as a result of } \\
\text { habitat quality. Habitat quality can be manipu- } \\
\text { lated through management; it is predicted that } \\
\text { viability of populations at the periphery of spe- } \\
\text { cies' range will be less resilient to marginal } \\
\text { habitat conditions }\end{array}$} \\
\hline $\begin{array}{l}\text { Southern periphery - optimal } \\
\text { habitat }\end{array}$ & $13 / 12$ & \\
\hline $\begin{array}{l}\text { South periphery - marginal habi- } \\
\text { tat }\end{array}$ & $15 / 13$ & \\
\hline Central range- optimal habitat & $15 / 11$ & \\
\hline Central range - marginal habitat & $17 / 15$ & \\
\hline $\begin{array}{l}\text { Northern/western periphery - } \\
\text { optimal habitat }\end{array}$ & $20 / 17$ & \\
\hline $\begin{array}{l}\text { Northern/western periphery - } \\
\text { marginal habitat }\end{array}$ & $23 / 20$ & \\
\hline Maximum age of reproduction & 60 & \\
\hline $\begin{array}{l}\text { Annual \% adult females repro- } \\
\text { ducing (S.D.) }\end{array}$ & & \multirow{3}{*}{$\begin{array}{l}\text { Annual proportion of females in a population } \\
\text { reproducing will be greater and less variable } \\
\text { from year to year in optimal habitat than mar- } \\
\text { ginal habitat. I chose less optimistic values } \\
\text { (even under "optimal" habitat) than the values } \\
\text { used in Miller et al. 2001. }\end{array}$} \\
\hline optimal habitat & $95(5)$ & \\
\hline marginal habitat & $80(10)$ & \\
\hline Maximum clutch size & 10 & \\
\hline Mean clutch size & & \multirow{4}{*}{$\begin{array}{l}\text { Mean clutch sizes are categorized according to } \\
\text { location within geographic range. Model con- } \\
\text { straints assume that clutch sizes do not vary } \\
\text { with age of female; although this assumption } \\
\text { may be violated, there are no data to estimate } \\
\text { age-specific fecundity. Individual variation } \\
\text { (due to body size) can be modeled by con- } \\
\text { straining distribution of clutch sizes within a } \\
\text { given year. Year to year variation in mean } \\
\text { clutch size will be reflected in S.D. }\end{array}$} \\
\hline Southern periphery & 7.5 & \\
\hline Central range & 6.75 & \\
\hline Northern/western periphery & 5 & \\
\hline Overall offspring sex ratio & 0.5 & \\
\hline $\begin{array}{l}\text { All adult males in breeding } \\
\text { pool? }\end{array}$ & Yes & \\
\hline$\%$ annual mortality (SD)* & & \multirow{6}{*}{$\begin{array}{l}\text { Annual mortalities are based on information } \\
\text { from the literature (incuding Miller et al. 2001) } \\
\text { and are assumed to be most influenced by } \\
\text { environmental stochasticity, modeled in the } \\
\text { S.D. There are very few data available in litera- } \\
\text { ture on which to base survivorship/mortality } \\
\text { estimates. Adult survivorship data are based } \\
\text { on long-term data from translocated popula- } \\
\text { tions (Ashton and Burke, 2007; Tuberville et } \\
\text { al., } 2008 \text { [in review]). }\end{array}$} \\
\hline hatchlings & 96 & \\
\hline yearlings & 55 & \\
\hline juveniles (age 2-4) & 25 & \\
\hline subadult & 3 & \\
\hline adult & 1.5 & \\
\hline
\end{tabular}


Table A2. Stable age distribution calculated by VORTEX ${ }^{\circledR}$ for an initial population of 100 individuals.

\begin{tabular}{|c|c|c|c|}
\hline Age & Males & Females & $\begin{array}{c}\text { Total no. } \\
\text { indiv. }\end{array}$ \\
\hline 1 & 5 & 5 & 10 \\
\hline 2 & 2 & 2 & 4 \\
\hline 3 & 1 & 1 & 2 \\
\hline 4 & 1 & 1 & 2 \\
\hline 5 & 1 & 1 & 2 \\
\hline 6 & 1 & 1 & 2 \\
\hline 7 & 1 & 1 & 2 \\
\hline 8 & 1 & 1 & 2 \\
\hline 9 & 1 & 1 & 2 \\
\hline 10 & 1 & 1 & 2 \\
\hline 11 & 1 & 1 & 2 \\
\hline 12 & 0 & 0 & 0 \\
\hline 13 & 1 & 1 & 2 \\
\hline 14 & 1 & 1 & 2 \\
\hline 15 & 1 & 1 & 2 \\
\hline 16 & 1 & 1 & 2 \\
\hline 17 & 0 & 0 & 0 \\
\hline 18 & 1 & 1 & 2 \\
\hline 19 & 1 & 1 & 2 \\
\hline 20 & 1 & 1 & 2 \\
\hline 21 & 0 & 1 & 1 \\
\hline 22 & 1 & 1 & 2 \\
\hline 23 & 1 & 0 & 1 \\
\hline 24 & 0 & 1 & 1 \\
\hline 25 & 1 & 1 & 2 \\
\hline 26 & 1 & 1 & 2 \\
\hline 27 & 0 & 1 & 1 \\
\hline 28 & 1 & 0 & 1 \\
\hline 29 & 1 & 1 & 2 \\
\hline 30 & 0 & 1 & 1 \\
\hline & & & \\
\hline
\end{tabular}

\begin{tabular}{|l|c|c|c|}
\hline Age & Males & Females & $\begin{array}{c}\text { Total no. } \\
\text { indiv. }\end{array}$ \\
\hline 31 & 1 & 1 & 2 \\
\hline 32 & 1 & 1 & 2 \\
\hline 33 & 0 & 0 & 0 \\
\hline 34 & 1 & 1 & 2 \\
\hline 35 & 0 & 1 & 1 \\
\hline 36 & 1 & 1 & 2 \\
\hline 37 & 0 & 1 & 1 \\
\hline 38 & 1 & 1 & 2 \\
\hline 39 & 1 & 0 & 1 \\
\hline 40 & 0 & 1 & 1 \\
\hline 41 & 1 & 1 & 2 \\
\hline 42 & 0 & 1 & 1 \\
\hline 43 & 1 & 1 & 2 \\
\hline 44 & 0 & 0 & 0 \\
\hline 45 & 1 & 1 & 2 \\
\hline 46 & 0 & 1 & 1 \\
\hline 47 & 1 & 1 & 2 \\
\hline 48 & 0 & 1 & 1 \\
\hline 49 & 1 & 1 & 2 \\
\hline 50 & 0 & 0 & 0 \\
\hline 51 & 1 & 1 & 2 \\
\hline 52 & 0 & 1 & 1 \\
\hline 53 & 0 & 1 & 1 \\
\hline 54 & 1 & 1 & 2 \\
\hline 55 & 0 & 1 & 1 \\
\hline 56 & 1 & 0 & 1 \\
\hline 57 & 0 & 1 & 1 \\
\hline 58 & 1 & 1 & 2 \\
\hline 59 & 0 & 1 & 1 \\
\hline 60 & 0 & 1 & 1 \\
\hline Total & 44 & 56 & 100 \\
\hline
\end{tabular}


Table A3. Manipulations of the baseline model for demographic sensitivity testing. The simulated population has an initial size of 100 individuals and occurs in optimal habitat in the central range. (The original parameter values used in the baseline model are represented by

"B.")

\begin{tabular}{|c|c|c|c|}
\hline Scenario name & Variable modified & $\begin{array}{l}\text { Relationship to } \\
\text { baseline }\end{array}$ & Numeric value \\
\hline ST.Scenario 17.max_longev(40) & MaxAgeRepr & B -20 & 40 \\
\hline ST.Scenario 17.max_longev(50) & MaxAgeRepr & B - 10 & 50 \\
\hline ST.Scenario 17.max_longev(70) & MaxAgeRepr & $B+10$ & 70 \\
\hline ST.Scenario 17.max_longev(80) & MaxAgeRepr & $B+20$ & 80 \\
\hline ST.Scenario 17.max_longev(100) & MaxAgeRepr & $B+40$ & 100 \\
\hline ST.Scenario 17.sex_ratio(1) & SexRatio & $\mathrm{B}^{*} .8$ & 40 \\
\hline ST.Scenario 17.sex_ratio(2) & SexRatio & $B * .9$ & 45 \\
\hline ST.Scenario 17.sex_ratio(3) & SexRatio & $B * 1.10$ & 55 \\
\hline ST.Scenario 17.sex_ratio(4) & SexRatio & $B * 1.2$ & 60 \\
\hline ST.Scenario 17.clutch_size(-2) & MeanProgenyPerYr & $B-2$ & 4.75 \\
\hline ST.Scenario 17.clutch_size(+2) & MeanProgenyPerYr & $\mathrm{B}+2$ & 8.75 \\
\hline ST.Scenario 17.adult_surv(+05) & AdultSurv & $B * .95$ & $+5 \%$ \\
\hline ST.Scenario 17.adult_surv(-05) & AdultSurv & $B * 1.05$ & $-5 \%$ \\
\hline ST.Scenario 17.adult_surv $(+10)$ & AdultSurv & $B * .90$ & $+10 \%$ \\
\hline ST.Scenario 17.adult_surv(-10) & AdultSurv & $B * 1.10$ & $-10 \%$ \\
\hline ST.Scenario 17.perc_repro(+10) & PercentBreed_Pop1 & $B * 1.10$ & $+10 \%$ \\
\hline ST.Scenario 17.perc_repro(-10) & PercentBreed_Pop1 & $B * .9$ & $-10 \%$ \\
\hline ST.Scenario 17.perc_repro(+20) & PercentBreed_Pop2 & $B * 1.2$ & $+20 \%$ \\
\hline ST.Scenario 17.perc_repro(-20) & PercentBreed_Pop3 & $B * .8$ & $-20 \%$ \\
\hline ST.Scenario 17.hatch_surv(x2) & Mortality_Age0 & $B * .5$ & $2 x$ \\
\hline ST.Scenario 17.hatch_surv(x5) & Mortality_Age0 & $B * .2$ & $5 x$ \\
\hline ST.Scenario 17.hatch_surv(x10) & Mortality_Age0 & $B * .1$ & $10 x$ \\
\hline ST.Scenario 17.hatch_surv $(+10)$ & Mortality_Age0 & $B * .9$ & $+10 \%$ \\
\hline ST.Scenario 17.hatch_surv(+20) & Mortality_Age0 & $\mathrm{B}^{*} .8$ & $+20 \%$ \\
\hline ST.Scenario 17.hatch_surv(+5) & Mortality_Age0 & $B * .95$ & $+5 \%$ \\
\hline ST.Scenario 17.hatch_surv(+1) & Mortality_Age0 & $B * .99$ & $+1 \%$ \\
\hline ST.Scenario 17.hatch_surv(+2) & Mortality_Age0 & $B * .98$ & $+2 \%$ \\
\hline ST.Scenario 17.hatch_surv(+3) & Mortality_Age0 & $B * .97$ & $+3 \%$ \\
\hline ST.Scenario 17.hatch_surv(+4) & Mortality_Age0 & $B * .96$ & $+4 \%$ \\
\hline ST.Scenario 17.yearling_surv(+10) & Mortality_Age1 & $B * .9$ & $+10 \%$ \\
\hline ST.Scenario 17.yearling_surv(+20) & Mortality_Age1 & $\mathrm{B}^{*} .8$ & $+20 \%$ \\
\hline ST.Scenario 17.yearling_surv(x2) & Mortality_Age1 & $B * .5$ & $2 x$ \\
\hline ST.Scenario 17.juv_surv(+10) & Mortality_Age2to4 & $B * .9$ & $+10 \%$ \\
\hline ST.Scenario 17.juv_surv(+20) & Mortality_Age2to4 & $\mathrm{B}^{*} .8$ & $+20 \%$ \\
\hline
\end{tabular}




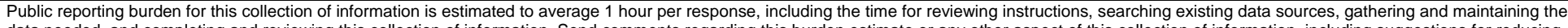

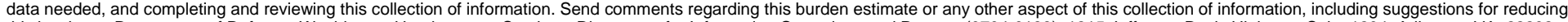

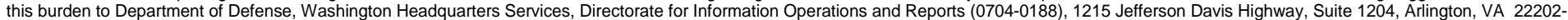

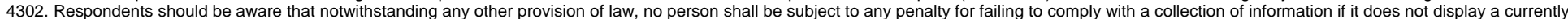
4302. Respondents should be aware that notwithstanding any other provision of law, no person shall bid OMB control number. PLEASE DO NOT RETURN YOUR FORM TO THE ABOVE ADDRESS.
valid
1. REPORT DATE (DD-MM-YYYY)
2. REPORT TYPE
3. DATES COVERED (From - To)

17-01-2009

Final

4. TITLE AND SUBTITLE

Estimating Viability of Gopher Tortoise Populations

5a. CONTRACT NUMBER

5b. GRANT NUMBER

5c. PROGRAM ELEMENT

6. AUTHOR(S)

Tracey D. Tuberville, J . Whitfield Gibbons, and Harold E. Balbach

5d. PROJECT NUMBER

622720A896

5e. TASK NUMBER

5f. WORK UNIT NUMBER

F61GB7

7. PERFORMING ORGANIZATION NAME(S) AND ADDRESS(ES)

U.S. Army Engineer Research and Development Center (ERDC)

Construction Engineering Research Laboratory (CERL)

PO Box 9005,

Champaign, IL 61826-9005

PERFORMING ORGANIZATION REPORT NUMBER

ERDC/ CERL TR-09-2

9. SPONSORING I MONITORING AGENCY NAME(S) AND ADDRESS(ES)

Environmental Division Office

10. SPONSOR/MONITOR'S ACRONYM(S)

600 Army Pentagon

Washington, DC 20310-1000

DAIM-ISE

11. SPONSOR/MONITOR'S REPORT NUMBER(S)

12. DISTRIBUTION / AVAILABILITY STATEMENT

Approved for public release; distribution is unlimited.

13. SUPPLEMENTARY NOTES

\section{ABSTRACT}

Although the gopher tortoise (Gopherus polyphemus) is widespread across the southeastern United States where conditions are favorable, the species is in serious decline. While biologists and land managers might consider any of a variety of in-situ and ex-situ management options when determining how best to manage individual populations, there are no good decision tools for evaluating or predicting whether the existing population is viable in the long term. This study developed demographic models for gopher tortoise populations and has proposed to use those models to predict outcomes for a variety of population conditions and management scenarios. The major limiting factor in the development of models was the availability of complete life-history data. Because of its demonstrated importance in population stability in other turtle species, adult survivorship was assumed to be high in all model scenarios. However, reproduction and survivorship of other life stages varied among simulations. The model results demonstrated that, of the parameters manipulated, hatchling survivorship was the most critical life history stage because of the very small likelihood that hatchlings survive to their second year. Any management actions that increase hatchling success are likely to increase population viability significantly.

15. SUBJECT TERMS

biological assessments

natural resource management

gopher tortoise land management

\begin{tabular}{l|l}
\hline 16. SECURITY CLASSIFICATION OF: & 17. LIMITATION
\end{tabular}

\begin{tabular}{|l|l|l|}
\hline \multicolumn{3}{|c|}{ a. REPORT } \\
Unclassified & $\begin{array}{c}\text { b. ABSTRACT } \\
\text { Unclassified }\end{array}$ & $\begin{array}{c}\text { c. THIS PAGE } \\
\text { Unclassified }\end{array}$ \\
\hline
\end{tabular}

OF ABSTRACT

SAR
18. NUMBER OF PAGES

60 19a. NAME OF RESPONSIBLE PERSON

19b. TELEPHONE NUMBER (include area code) 